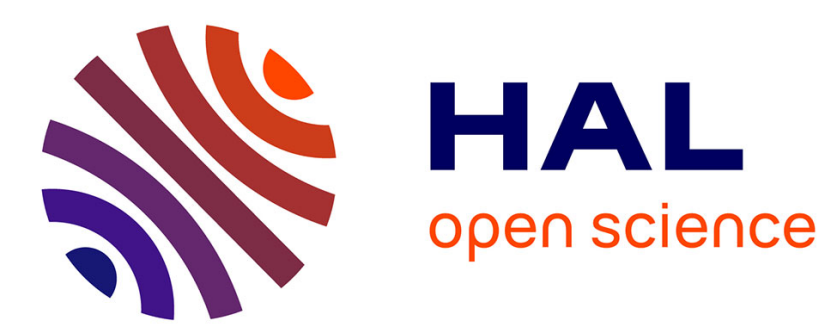

\title{
Internal Coalgebras in Cocomplete Categories: Generalizing the Eilenberg-Watts Theorem
}

\author{
Laurent Poinsot, Hans-E Porst
}

\section{To cite this version:}

Laurent Poinsot, Hans-E Porst. Internal Coalgebras in Cocomplete Categories: Generalizing the Eilenberg-Watts Theorem. Journal of Algebra and Its Applications, In press. hal-02510509

\author{
HAL Id: hal-02510509 \\ https://hal.science/hal-02510509
}

Submitted on 17 Mar 2020

HAL is a multi-disciplinary open access archive for the deposit and dissemination of scientific research documents, whether they are published or not. The documents may come from teaching and research institutions in France or abroad, or from public or private research centers.
L'archive ouverte pluridisciplinaire HAL, est destinée au dépôt et à la diffusion de documents scientifiques de niveau recherche, publiés ou non, émanant des établissements d'enseignement et de recherche français ou étrangers, des laboratoires publics ou privés. 


\title{
Internal Coalgebras in Cocomplete Categories: Generalizing the Eilenberg-Watts-Theorem
}

\author{
Laurent Poinsot \\ LIPN, UMR CNRS 7030, University Sorbonne Paris North, \\ and CREA, École de l'Air, France. \\ laurent.poinsot@lipn.univ-paris13.fr \\ Hans-E. Porst* \\ Department of Mathematical Sciences, University of Stellenbosch, \\ Stellenbosch, South Africa. \\ porst@math.uni-bremen.de
}

\begin{abstract}
The category of internal coalgebras in a cocomplete category $\mathcal{C}$ with respect to a variety $\mathcal{V}$ is equivalent to the category of left adjoint functors from $\mathcal{V}$ into $\mathcal{C}$. This can be seen best when considering such coalgebras as finite coproduct preserving functors from $\mathcal{T}_{\mathcal{V}}^{\text {op }}$, the dual of the Lawvere theory of $\mathcal{V}$, into $\mathcal{C}$ : coalgebras are restrictions of left adjoints and any such left adjoint is the left Kan extension of a coalgebra along the embedding of $\mathcal{T}_{\mathcal{V}}^{\text {op }}$ into Alg $\mathcal{T}$. Since ${ }_{S} \operatorname{Mod}$-coalgebras in the variety ${ }_{R} \operatorname{Mod}$ for rings $R$ and $S$ are nothing but left $S$-, right $R$-bimodules, the equivalence above generalizes the Eilenberg-Watts Theorem and all its previous generalizations. Generalizing and strengthening Bergman's completeness result for categories of internal coalgebras in varieties we also prove that the category of coalgebras in a locally presentable category $\mathcal{C}$ is locally presentable and comonadic over $\mathcal{C}$ and, hence, complete in particular. We show, moreover, that Freyd's canonical constructions of internal coalgebras in a variety define left adjoint functors. Special instances of the respective right adjoints appear in various algebraic contexts and, in the case where $\mathcal{V}$ is a commutative variety, are coreflectors from the category $\operatorname{Coalg}(\mathcal{T}, \mathcal{V})$ into $\mathcal{V}$.
\end{abstract}

MSC 2010: Primary 18D35, Secondary 08C5

Keywords: Internal coalgebra, Lawvere theory, (commutative) variety, locally presentable category.

\section{Introduction}

The Eilenberg-Watts [14, 29] Theorem states that the only additive and colimit preserving functors $L:{ }_{R} \operatorname{Mod} \rightarrow{ }_{S} M o d$ between the categories of left modules over not necessarily commutative rings $R$ and $S$ are the functors $M_{R} \otimes_{R}$ - for left $S$-, right $R$-bimodules ${ }_{S} M_{R}$. By the (dual of) the Special Adjoint Functor Theorem this is equivalent to saying that these tensoring functors are the only additive and left adjoint functors between module categories. Since equivalences between

*Permanent address: Department of Mathematics, University of Bremen, 28359 Bremen, Germany. 
categories are adjunctions, this result is of importance for describing classical Morita theory of rings, one of the early reasons for the interest in it.

Decades later is has been felt necessary to generalize this to additive left adjoint functors $L:{ }_{R} \operatorname{Mod} \rightarrow \mathcal{C}$ for categories $\mathcal{C}$ more general than module categories. The most recent generalization [23] assumes $\mathcal{C}$ to be a cocomplete additive and $k$-linear category and $R$ the underlying ring of a $k$-algebra (for some commutative ring $k$ ) in order to prove a non-trivial result about quasi-coherent sheaves.

The main problem, obviously, then is to replace the concept of bimodule by a more general one. In [11 and 23] this is achieved by choosing left $R$-objects in $\mathcal{C}$ (see Section 2.1.3 below). The shortcomings of this approach are that this idea neither can be further generalized to nonadditive categories $\mathcal{C}$ as codomains for $L$ nor to varieties other then module categories as domains of $L$. Note that the Eilenberg-Watts Theorem can, alternatively, be read as characterizing the additive right adjoint functors $K:{ }_{S} M o d \rightarrow{ }_{R} M o d$ as the functors $\operatorname{Hom}_{S}\left({ }_{S} M,-\right)$ for left $S$-, right $R$-bimodules ${ }_{S} M_{R}$, and that these the only functors making the following diagram commute:

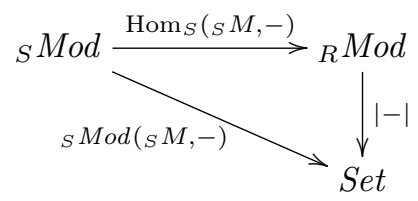

The functors $\operatorname{Hom}_{S}\left({ }_{S} M,-\right)$, thus, are examples $\mathcal{V}$-representable functors in the sense of Freyd [16], that is, of functors making the following diagram commute, where $\mathcal{C}$ is a category with finite coproducts and $|-|$ denotes the forgetful functor of a variety $\mathcal{V}$.

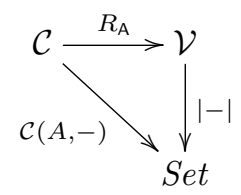

Such functors provide an equivalent way of describing, for any variety $\mathcal{V}$, internal $\mathcal{V}$-coalgebras in a category $\mathcal{C}$ with finite coproducts, that is, internal $\mathcal{V}$-algebras in the dual of a category $\mathcal{C}$ with finite products. The latter notion is essentially obtained by translating the concept of equationally defined algebras from the language of universal algebra into that of category theory, where satisfaction of an equational algebra axiom is expressed by commutativity of a diagram.

Consequently, the ${ }_{R} M o d$-coalgebras in ${ }_{S} M o d$ are precisely the left $S$-, right $R$-bimodules, an observation Freyd - somewhat surprisingly - did not notice, and this is the appropriate generalization of bimodules needed for generalizing the Eilenberg-Watts theorem.

As gradually became clear, the concept of internal coalgebras provides a common perspective on apparently completely unrelated concepts, constructions and results as, for example, the construction of homotopy groups, the change of rings functors in module theory, the Eilenberg-Watts theorem as mentioned above, or the group-like and primitive elements functors of Hopf algebra theory.

Freyd in his paper initiated a systematic study of these structures by what one may call a semi-categorical approach and introduced canonical ways of constructing coalgebras in a variety. Somewhat later, without reference to these papers, internal coalgebras in the category of commutative rings were introduced and applied in 28. More recently gaps in this theory were closed and non trivial results added by Bergmann [7; most notably, he proved that the category 
of $\mathcal{V}$-coalgebras in a variety $\mathcal{W}$ is complete and its forgetful functor into $\mathcal{W}$ has a right adjoint. However, his use of algebraic methods only requires a considerable amount of unusual technicalities. So, from its very beginning, the study of internal coalgebras appears as an interplay between algebraic and category theoretic concepts and methods.

It is the intention of this note to show that a purely categorical approach - besides providing the appropriate setting for generalizing the Eilenberg-Watts Theorem - simplifies the presentation of the theory of coalgebras and leads to new results, improvements of known ones, and further applications. For the sake of those readers not too familiar with categorical methods, we include translations of crucial concepts and results into the language of universal algebra.

We, hence, consider coalgebras in a category $\mathcal{C}$ with finite coproducts simply as functors $\mathcal{T}^{\text {op }} \stackrel{\text { A }}{\rightarrow} \mathcal{C}$ from the dual $\mathcal{T}^{\text {op }}$ of a Lawvere theory into $\mathcal{C}$ which preserve finite coproducts. This approach becomes particularly convincing, when $\mathcal{C}$ not only has finite coproducts but even is cocomplete. Then there results quite obviously an equivalence of coalgebras and left adjoints, which generalizes the Eilenberg-Watts Theorem and can be expressed conceptually as follows: Up to natural equivalence, $\mathcal{T}$-coalgebras $\mathcal{T}^{\text {op }} \stackrel{\mathrm{A}}{\rightarrow} \mathcal{C}$ are nothing but the restrictions of left adjoints Alg $\mathcal{T} \stackrel{L}{\rightarrow} \mathcal{C}$ to $\mathcal{T}^{\text {op }}$, considered as a subcategory of $\operatorname{Alg} \mathcal{T}$, while the left adjoint $L_{\mathrm{A}}$ corresponding to a coalgebra $A$ is characterized by any of the following universal properties, where the first one is purely categorical in nature while the second one is in view of Section 1.2 .4 below of an algebraic flavour.

Kan $L_{\mathrm{A}}$ is the left Kan extension of A along the embedding $\mathcal{T}^{\mathrm{op}} \stackrel{Y}{\rightarrow} \operatorname{Alg} \mathcal{T}$.

Sift $L_{\mathrm{A}}$ is the sifted colimit preserving extension of $\mathrm{A}$.

Concerning improvements of the theory of coalgebras we show for example:

- For every Lawvere theory $\mathcal{T}$ the category $\operatorname{Coalg}(\mathcal{T}, \mathcal{C})$ of $\mathcal{T}$-coalgebras in a locally presentable category $\mathcal{C}$ is again a locally presentable category and, hence, complete in particular. This generalizes considerably Bergman's result in [7. Moreover, its underlying functor into Coalg $(\mathcal{T}, \mathcal{C}) \stackrel{\|-\|}{\longrightarrow} \mathcal{C}$ has a right adjoint (as shown in [7] for $\mathcal{C}$ being an arbitrary variety, while the paper 13 claims to present a proof for corings in the category of rings (see however its review)), and, moreover, is comonadic (as shown for $\mathcal{C}=$ Set by Isbell [18]). However, not each such comonadic category is of the form $\operatorname{Coalg}(\mathcal{T}, \mathcal{C})$.

- The canonical constructions of coalgebras in a variety already mentioned by Freyd are generalized to coalgebras in a locally presentable category. Their existence in the case of varieties also becomes immediately evident by our categorical approach, as is the fact that they are left adjoint functors. The respective right adjoints can be viewed as a generalization of both, the group-like elements and the primitive elements functors, known from Hopf algebra theory.

In the case of a commutative variety $\mathcal{V}$ with Lawvere theory $\mathcal{T}_{\mathcal{V}}$ the canonical construction provides a full coreflective embedding of $\mathcal{V}$ into $\operatorname{Coalg}\left(\mathcal{T}_{\mathcal{V}}, \mathcal{V}\right)$. We show by examples that these embeddings may or may not be equivalences.

We also show that the theory developed can be of use (besides generalizing the EilenbergWatts Theorem); we show for example how the following, familiar algebraic results are obtained easily. 
- The General linear group and Special linear group functors $G L_{n}, S L_{n}:$ Ring $\rightarrow$ Grp have left adjoints.

- For any ring homomorphism $\phi: R \rightarrow S$ the Extension of scalars-functor ${ }_{R} \operatorname{Mod} \rightarrow{ }_{S}$ Mod is left adjoint to the Restriction of scalars-functor ${ }_{S} \operatorname{Mod} \rightarrow{ }_{R} \operatorname{Mod}$ and this is left adjoint to the Coextension of scalars-functor ${ }_{R} \operatorname{Mod} \rightarrow{ }_{S}$ Mod.

- Each symmetric algebra, that is, each free commutative $R$-algebra $\Sigma_{*}(M)$ over an $R$-module $M$, is a Hopf algebra whose set of primitive elements contains $M$.

- Moreover we show that our results provide a natural setting for Morita equivalence of arbitrary varieties, generalizing classical Morita theory for module categories.

The paper is organized as follows:

Section 1 is mainly devoted to an introduction to the concept of Lawvere theories. In its part on varieties we improve Freyd's approach to the interpretation of terms in a category with finite products, necessary in this context. What seems to be new is the result that the sifted colimit preserving extension of a functor $\mathcal{T}^{\mathrm{op}} \rightarrow \mathcal{C}$ is nothing but its left Kan extension along the embedding $\mathcal{T}^{\mathrm{op}} \hookrightarrow \operatorname{Alg} \mathcal{T}$.

Section 2 deals with the general theory of internal coalgebras. Subsection 2.1 provides the various possible definitions and proofs of their equivalence, while subsections 2.3 and 2.2 deal with the properties of the categories of internal coalgebras and provide a first set of examples.

Section 3 develops the theory the categories of coalgebras in varieties. Subsection 3.1 discusses the canonical construction of coalgebras on free algebras and its adjunction; it closes with an application of these results in the context of Kan's construction of cogroups in the category of groups. Subsection 3.1.2 provides some non-trivial applications. Subsection 3.2 deals with coalgebras in commutative varieties. We show in particular, that the canonical construction just mentioned, in this case extends to a full coreflective embedding.

\section{Preliminaries}

\subsection{Some concepts and notations}

We next introduce some concepts and notations not every reader may be familiar with.

Semi-additive categories. A category $\mathcal{C}$ is called semi-additive, if it has finite biproducts. Every such category is enriched over ${ }_{c}$ Mon. If $\mathcal{C}$ even is enriched over $A b$ it is called additive.

Locally presentable categories. Given a regular cardinal $\lambda$, an object $P$ in a category $\mathcal{C}$ is called $\lambda$ presentable iff $\mathcal{C}(P,-)$ preserves $\lambda$-filtered colimits. A category $\mathcal{C}$ is called locally presentable, provided that it is cocomplete and has, for some $\lambda$, a set of $\lambda$-presentable objects of which each $\mathcal{C}$-objects is a $\lambda$-filtered colimit. Every locally presentable category is complete, wellpowered and cowellpowered, and has certain factorization structures of morphisms (see [2] for details).

Examples of such categories are all varieties and quasivarieties, the category of partially ordered sets and, more generally, every universal Horn class, and the category of small categories. Also, for locally presentable categories $\mathcal{C}$ and $\mathcal{D}$, the category $\operatorname{Ladj}(\mathcal{D}, \mathcal{C})$ of left adjoint functors $\mathcal{D} \rightarrow \mathcal{C}$ is locally presentable (see $[9]$ ). 
Functor coalgebras. The category Coalg $F$ of $F$-algebras in $\mathcal{C}$ for a functor $\mathcal{C} \stackrel{F}{\rightarrow} \mathcal{C}$ has as objects all pairs $(C, C \stackrel{\gamma}{\rightarrow} F C)$ and as morphisms $(C, \gamma) \stackrel{f}{\rightarrow}\left(C^{\prime}, \gamma^{\prime}\right)$ those $\mathcal{C}$-morphisms $C \stackrel{f}{\rightarrow} C^{\prime}$ which satisfy $F f \circ \gamma=\gamma^{\prime} \circ f$. The obvious forgetful functor Coalg $F \rightarrow \mathcal{C}$ reflects isomorphisms and creates colimits and those limits which are preserved by $F$.

Kan extensions. Given functors $\mathcal{B} \stackrel{F}{\rightarrow} \mathcal{C}$ and $\mathcal{B} \stackrel{Y}{\rightarrow} \mathcal{D}$ on small category $\mathcal{B}$ a left Kan extension of $F$ along $Y$ is a functor $\operatorname{Lan}_{Y} F: \mathcal{D} \rightarrow \mathcal{C}$ together with a natural transformation $\alpha: F \Rightarrow$ $\operatorname{Lan}_{Y} F \circ Y$ such that for any other such pair $(G: \mathcal{D} \rightarrow \mathcal{C}, \beta: F \Rightarrow G \circ Y) \beta$ factors uniquely through $\alpha . \alpha$ is a natural isomorphism, if $Y$ is full and faithful; if $Y$ is a full embedding one even has $\alpha=i d$. $\operatorname{Lan}_{Y} F$ can be constructed with $\operatorname{Lan}_{Y} F$ acting on a $\mathcal{D}$-object $D$ as the colimit of the diagram $Y \downarrow D \stackrel{\Phi_{D}}{\longrightarrow} \mathcal{B} \stackrel{F}{\rightarrow} \mathcal{C}$ where $\Phi_{D}$ is the obvious forgetful functor into $\mathcal{B}$, if these colimits exist in $\mathcal{C}$ for each $D$ (see e.g. [22, Chapter X.3]).

In particular, if the Yoneda embedding $Y_{\mathcal{B}^{\text {op }}}$ factors as $\mathcal{B}^{\text {op }} \stackrel{Y}{\rightarrow} \mathcal{A} \stackrel{I}{\hookrightarrow} S e t^{\mathcal{B}}$ for some full subcategory $\mathcal{A}$ of $S e t^{\mathcal{B}}$ and $\mathcal{C}$ has $Y \downarrow A$-colimits for each $A$ in $\mathcal{A}$, one has natural isomorphisms

$$
\mathcal{C}\left(\operatorname{Lan}_{Y} F(A), C\right) \simeq \operatorname{Set}^{\mathcal{B}}\left(A, \hat{R}_{F} C\right)
$$

where $\hat{R}_{F}: \mathcal{C} \rightarrow S e t^{\mathcal{B}}$ is the functor with $\hat{R}_{F} C=\mathcal{B} \stackrel{F^{\mathrm{op}}}{\longrightarrow} \mathcal{C}^{\mathrm{op}} \stackrel{\mathcal{C O p}^{\mathrm{op}}(C,-)=\mathcal{C}(-, C)}{\longrightarrow}$ Set for each $\mathcal{C}$-object $C$. Thus, the Yoneda extension $\operatorname{Lan}_{Y_{\mathcal{B} \text { op }} F}$ has $\hat{R}_{F}$ as its right adjoint.

Sifted colimits. A small category $\mathcal{D}$ is called sifted if finite products in Set commute with colimits over $\mathcal{D}$. Colimits of diagrams over sifted categories are called sifted colimits. Sifted colimits in a variety are precisely those colimits which are - like all limits - created by the forgetful functor. See [4] for more details on this type of colimits.

Equifiers. Given an $I$-indexed family of pairs of functors $F^{i}, G^{i}: \mathcal{C} \rightarrow \mathcal{C}_{i}$ and for each $i \in I$ a pair of natural transformations $\phi^{i}, \psi^{i}: F^{i} \Rightarrow G^{i}$, we call (following [2]) the full subcategory of $\mathcal{C}$ spanned by all objects $C$ satisfying $\phi_{C}^{i}=\psi_{C}^{i}$ for all $i \in I$ the joint equifier $\operatorname{Equ}\left(\phi^{i}, \psi^{i}\right)_{i}$ of the family $\left(\phi^{i}, \psi^{i}\right)$.

Some notations. The hom-functors of a category $\mathcal{C}$ are denoted by $\mathcal{C}(C,-)$. The equalizer of a parallel pair $(f, g)$ in $\mathcal{C}$ is denoted by $\operatorname{Eq}_{\mathcal{C}}(f, g)$. If $\left(P,\left(P \stackrel{\pi_{i}}{\longrightarrow} X_{i}\right)_{i}\right)$ is a product and $\left(X \stackrel{f_{i}}{\longrightarrow} X_{i}\right)_{i}$ a family of morphisms in a category $\mathcal{C}$ we denote by $X \stackrel{\left\langle\left(f_{i}\right)_{i}\right\rangle}{\longrightarrow} P$ the unique $\mathcal{C}$-morphism with $\pi_{i} \circ\left\langle\left(f_{i}\right)_{i}\right\rangle=f_{i}$ for each $i$. The $X$-fold copower of an object $C$ is denoted $X \cdot C$. Dually, given a coproduct $\left(C,\left(X_{i} \stackrel{\mu_{i}}{\longrightarrow} C\right)_{i}\right)$, by $C \stackrel{\left[\left(f_{i}\right)_{i}\right]}{\longrightarrow} X$ we denote the unique morphism induced by a family of morphisms $\left(X_{i} \stackrel{f_{i}}{\longrightarrow} X\right)_{i}$. Functor categories are denoted by $\mathcal{B}^{\mathcal{A}}$ or $[\mathcal{A}, \mathcal{B}]$. If, for some category $\mathcal{C}$, there is a category $\mathcal{A}(\mathcal{C})$ of "structured $\mathcal{C}$-objects" with forgetful functor $\mathcal{A}(\mathcal{C}) \stackrel{|-|}{\longrightarrow} \mathcal{C}$ we will occasionally, by slight abuse of notation, denote an object in $\mathcal{A}(\mathcal{C})$ with underlying $\mathcal{C}$-object $C$ simply by $\mathrm{C}$.

We use the following notation for some frequently used categories: Set denotes the category of sets, $\operatorname{Grp}(A b)$, that of (abelian) groups, Mon ( $\left.{ }_{c} M o n\right)$, that of (commutative) monoids, Ring, that of unital rings, ${ }_{R} \operatorname{Mod}\left(\operatorname{Mod}_{R}\right)$ that of left (right) $R$-modules, $A l g_{R}\left({ }_{c} A l g_{R}\right)$, that of (commutative) $R$-algebras over a commutative ring.

For $n \in \mathbb{N}$ we often denote an $n$-element set simply by $n$. 


\section{$1.2 \quad$ Varieties}

\subsubsection{Varieties by signatures}

A signature is a pair $\Sigma=(\bar{\Sigma}, \bar{\Sigma} \stackrel{a r}{\longrightarrow} \mathbb{N})$ consisting of a set $\bar{\Sigma}$ and an arity map $\bar{\Sigma} \stackrel{a r}{\longrightarrow} \mathbb{N}$. By $\left(\Sigma_{n}\right)_{n}$ we denote the family of preimages of $n$ under $a r$. In the sequel we usually don't distinguish notationally between $\bar{\Sigma}$ and $\Sigma$. Alg $\Sigma$ then is the category of $\Sigma$-algebras $\mathrm{A}=\left(A,\left(A^{\text {ar } \sigma} \stackrel{\sigma^{\mathrm{A}}}{\longrightarrow} A\right)_{\sigma \in \bar{\Sigma}}\right)$ and $T(\Sigma)_{n}$ the set of $\Sigma$-terms in $n$ variables in the sense of universal algebra. $T(\Sigma)$, the disjoint union of all sets $T(\Sigma)_{n}$, is the set of $\Sigma$-terms.

The interpretation of a term $t \in T(\Sigma)_{n}$ on a $\Sigma$-algebra $\mathrm{A}$ will be denoted by $A^{n} \stackrel{t^{\mathrm{A}}}{\rightarrow} A$. Since the familiar definitions of $t^{\mathrm{A}}$ are either given element-wise or use freeness of the $\Sigma$-algebras of $\Sigma$-terms and none of these methods works for arbitrary internal algebras in a category $\mathcal{C}$ with finite products, Freyd [16] suggested a recursive definition using products, which however has some shortcomings as already observed in its review. We therefore use the following recursive definition of the interpretation $A^{n} \stackrel{t^{\mathrm{A}}}{\rightarrow} A$ of a $\Sigma$-term $t \in T(\Sigma)_{n}$ in a $\Sigma$-algebra $\mathrm{A}$, which then will make sense in any category $\mathcal{C}$ with finite products in Section 1.2 .5 below, where for the fundamental terms, that is the "constants" $\sigma \in \Sigma_{0}$ and the "variables" $x_{1}, \ldots, x_{n}, \ldots$ we add a subscript $n$ to indicate that they are considered as elements of $T(\Sigma)_{n}$ (and not of some $T(\Sigma)_{m}$ with $m>n$ ) in order to avoid ambiguity:

1. $x_{n, j}^{\mathrm{A}}:=A^{n} \stackrel{\pi_{j}}{\longrightarrow} A$ is the $j^{t h}$ product projection, for the variables $x_{1}, \ldots, x_{n}$.

2. $t^{\mathrm{A}}:=A^{n} \stackrel{\left\langle\left(t_{i}^{\mathrm{A}}\right)_{i}\right\rangle}{\longrightarrow} A^{\operatorname{ar}(\tau)} \stackrel{\tau^{\mathrm{A}}}{\longrightarrow} A$, if $t=\tau\left(t_{1}, \cdots, t_{a r(\tau)}\right)$ with $t_{i} \in T(\Sigma)_{n}$. In particular

(a) $\sigma_{n}^{\mathrm{A}}:=A^{n} \stackrel{!}{\rightarrow} 1 \stackrel{\sigma^{\mathrm{A}}}{\rightarrow} A$ for each $t=\sigma \in \Sigma_{0}$, where! denotes the unique morphism,

(b) $\sigma\left(x_{1}, \ldots, x_{n}\right)^{\mathrm{A}}=A^{n} \stackrel{\sigma^{\mathrm{A}}}{\longrightarrow} A$ for each $\sigma \in \Sigma_{n}$ and $n>0$.

Since every homomorphism $\mathrm{A} \stackrel{f}{\rightarrow} \mathrm{A}^{\prime}$ satisfies $t^{\mathrm{A}^{\prime}} \circ f^{n}=f \circ t^{\mathrm{A}}$ for every $t \in T(\Sigma)_{n}$, every such $t$ determines a natural transformation $\tilde{t}:|-|^{n} \Rightarrow|-|$ with components $\tilde{t}_{\mathrm{A}}=t^{\mathrm{A}}$, where

$\operatorname{Alg} \Sigma \stackrel{|-|}{\longrightarrow}$ Set denotes the forgetful functor. By the Yoneda lemma the assignment $t \mapsto \tilde{t}$ defines a bijection between $T(\Sigma)_{n}$ and the set of natural transformations nat $\left(|-|^{n},|-|\right)$, since $|-|^{n}$ is represented by the free $\Sigma$-algebra on $n$ whose carrier set is $T(\Sigma)_{n}$. A set of $\Sigma$-equations is a family $\mathfrak{E}=\left(\mathfrak{E}_{n}\right)_{n}$ with $\mathfrak{E}_{n} \subset T(\Sigma)_{n} \times T(\Sigma)_{n}$. As usual we denote pairs $(s, t) \in \mathfrak{E}$ by $s=t$. A $\Sigma$-algebra $\mathrm{A}$ is said to satisfy the equation $s=t$ iff $t^{\mathrm{A}}=s^{\mathrm{A}}$. $\operatorname{Alg}(\Sigma, \mathfrak{E})$ denotes the full subcategory of $\operatorname{Alg} \Sigma$ of algebras satisfying all equations in $\mathfrak{E}$ and is called a variety. The pair $(\Sigma, \mathfrak{E})$ is occasionally called an equational theory.

Writing $t=\tau\left(t_{1}, \ldots t_{m}\right)$ with $\tau \in \Sigma_{m}$ and $t_{i} \in T(\Sigma)_{k}$ and $s=\sigma\left(s_{1}, \ldots s_{n}\right)$ with $\sigma \in \Sigma_{n}$ and $s_{j} \in T(\Sigma)_{k}$ a $\Sigma$-algebra A satisfies $s=t$ if and only if the following diagram commutes.

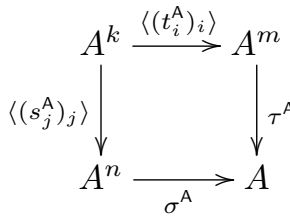


Satisfaction of an equational axiom $s=t$, thus, is the requirement for Diagram (4) to commute. Since the paths of this diagram are the components of the natural transformations $\tilde{t}$ and $\tilde{s}$, the variety $\operatorname{Alg}(\Sigma, \mathfrak{E})$ is, as a subcategory of $\operatorname{Alg} \Sigma$, the joint equifier $\operatorname{Equ}(\tilde{s}, \tilde{t})_{s=t \in \mathfrak{E}}$.

\subsubsection{Varieties by Lawvere theories}

The categorification of these notions is due to Lawvere [20], as is well known: Pairs $(\Sigma, \mathfrak{E})$ are substituted by Lawvere theories $\mathcal{T}$ and varieties by the full subcategories $A l g \mathcal{T}$ of $S e t^{\mathcal{T}}$ consisting of all finite product preserving functors. For the convenience of the reader not already familiar with the concept of Lawvere theory we add the following remarks.

Definition 1 A Lawvere theory is a category $\mathcal{T}$ with a countable set of objects $T_{0}, T_{1}, \ldots, T_{n}, \ldots$ (occasionally written simply as $0,1, \ldots, n, \ldots$ ) such that $\mathcal{T}$ has finite products and the object $T_{1}$ allows for each $n \in \mathbb{N}$ a specified product $\left(T_{n},\left(T_{n} \stackrel{\pi_{n, i}}{\longrightarrow} T_{1}\right)_{1 \leq i \leq n}\right)$. Lawvere theories and finite product preserving functors constitute the category Lawv.

The category $\operatorname{Alg} \mathcal{T}$ of $\mathcal{T}$-algebras has its objects all finite product preserving functors $\mathcal{T} \stackrel{A}{\rightarrow}$ Set and its morphisms the natural transformations between those. Evaluation at $T_{1}$ defines a faithful functor $\operatorname{Alg} \mathcal{T} \stackrel{|-|}{\longrightarrow}$ Set.

We recall the following basic facts of this categorification as follows, introducing some notations used in this note at the same time. For details we refer to [4].

I. The dual of a skeleton of the category of finite sets is a Lawvere theory $\mathcal{N}$. This is the initial object of Lawv. For any Lawvere theory $\mathcal{T}$ we denote by $T$ the unique Lawv-morphism $\mathcal{N} \rightarrow \mathcal{T}$. There is a forgetful functor from Lawv into the category of signatures acting as $\mathcal{T} \rightarrow \mathcal{T}(n, 1)=: \Sigma_{\mathcal{T}}$. This functor has a left adjoint which assigns to a signature $\Sigma$ the free theory $\mathcal{T}_{\Sigma}$ over $\Sigma$. In particular, $\Sigma_{\mathcal{T}_{\Sigma}}=T(\Sigma)$. There is a concrete isomorphism

$$
\operatorname{Alg} \mathcal{T}_{\Sigma} \simeq \operatorname{Alg} \Sigma
$$

given on objects by $\mathrm{A} \mapsto\left(\mathrm{A}\left(T_{1}\right),\left(\mathrm{A}\left(\sigma\left(x_{1}, \cdots, x_{a r \sigma}\right)\right)\right)_{\sigma \in \Sigma}\right)$.

II. There exists a dual biequivalence between Lawvere theories and varieties. In more detail:

(a) For every variety $\mathcal{V}=\operatorname{Alg}(\Sigma, \mathfrak{E})$ there exists an essentially unique Lawvere theory $\mathcal{T}_{\mathcal{V}}$ such that $\mathcal{V}$ is concretely equivalent to $\operatorname{Alg} \mathcal{T}_{\mathcal{V}}$. $\mathcal{T}_{\mathcal{V}}$ can be chosen to be the dual of the full subcategory $\left\langle F_{\mathcal{V}} n \mid n \in \mathbb{N}\right\rangle$ of $\mathcal{V}$ formed by choosing, for each $n \in \mathbb{N}$, a free $\mathcal{V}$-algebra $F_{\mathcal{V}} n$ in such a way that $F_{\mathcal{V}} n \neq F_{\mathcal{V}} m$ for $n \neq m$. Equivalently, $\mathcal{T}_{\mathcal{V}}(n, 1)$ is the set of natural transformations $|-|^{n} \Rightarrow|-|$. In other words, $\mathcal{T}_{\mathcal{V}}^{\text {op }}$ can be considered as a full subcategory of $\operatorname{Alg} \mathcal{T}_{\mathcal{V}}$.

(b) For every Lawvere theory $\mathcal{T}$ the category $\operatorname{Alg} \mathcal{T}$ is concretely equivalent to some variety $\mathcal{V}=\operatorname{Alg}(\Sigma, \mathfrak{E})$, equivalently, $\mathcal{T}$ is isomorphic to $\mathcal{T}_{\mathcal{V}}$ for some $\mathcal{V}$.

(c) Every morphism $\mathcal{S} \stackrel{\Phi}{\rightarrow} \mathcal{T}$ of Lawvere theories, that is, a finite product preserving functor, induces the algebraic functor $\operatorname{Alg} \mathcal{T} \stackrel{\Phi^{*}}{\longrightarrow} \operatorname{Alg} \mathcal{S}$ given by $(\mathcal{T} \stackrel{\mathrm{A}}{\rightarrow}$ Set $) \mapsto(\mathcal{S} \stackrel{\Phi}{\rightarrow}$ $\mathcal{T} \stackrel{\text { A }}{\rightarrow}$ Set). This is a concrete functor admitting a left adjoint $\Phi_{*}$. Every concrete functor $\mathcal{V} \stackrel{U}{\rightarrow} \mathcal{W}$ between varieties has a left adjoint $L_{U}$ and so determines a morphism in Lawv: Take the dual of the restriction $\left\langle F_{\mathcal{W}} n \mid n \in \mathbb{N}\right\rangle \stackrel{L_{U}}{\longrightarrow}\left\langle F_{\mathcal{V}} n \mid n \in \mathbb{N}\right\rangle$. 
In particular, for any Lawvere theory $\mathcal{T}, \operatorname{Alg} \mathcal{T} \stackrel{T^{*}}{\longrightarrow} \operatorname{Alg} \mathcal{N} \simeq S e t$ is the forgetful functor; it creates limits and sifted colimits and reflects isomorphisms. Its left adjoint $T_{*}$ is the free algebra functor.

III. Every Lawvere theory $\mathcal{T}$ is a regular quotient of a free one. In more detail: If Alg $\mathcal{T}$ is concretely isomorphic to the variety $\operatorname{Alg}(\Sigma, \mathfrak{E})$, then there exists a quotient morphism $\mathcal{T}_{\Sigma} \stackrel{[-]}{\longrightarrow} \mathcal{T}$ in Lawv. This functor identifies terms $s$ and $t$, whenever $(s, t)$ is deducible from $\mathfrak{E}$, that is, if every algebra in $\operatorname{Alg}(\Sigma, \mathfrak{E})$ satisfies the equation $(s, t)$. The functor $\operatorname{Alg} \mathcal{T} \stackrel{[-]^{*}}{\longrightarrow}$ $\operatorname{Alg} \mathcal{T}_{\Sigma}$ is a full embedding. In the language of universal algebra this quotient morphism corresponds to the canonical quotients $F_{\Sigma} n \stackrel{[-]}{\longrightarrow} F_{\mathcal{V}} n$ for each $n$ and, correspondingly, for each $\sigma \in \Sigma_{n}$, the $\mathcal{T}$-morphism $T_{n} \stackrel{[\sigma]}{\longrightarrow} T_{1}$ can, in view of II.(a) above, be identified with the $\mathcal{V}$-homomorphism $F_{\mathcal{V}} 1 \stackrel{\bar{\sigma}}{\rightarrow} F_{\mathcal{V}} n$ mapping 1 to $\left[\sigma\left(x_{1}, \ldots, x_{n}\right)\right]$.

IV. There are essentially one-to-one correspondences $\mathcal{T} \mapsto \mathbb{T}$ between Lawvere theories and finitary monads on Set, such that $\operatorname{Alg} \mathcal{T} \simeq S e t^{\mathbb{T}}$, and the functors $\Phi^{*}$ induced by theory morphisms and monad morphisms, respectively. The monad $\mathbb{T}$ is given by the adjunction $-\cdot F 1 \dashv \operatorname{Alg} \mathcal{T}(F 1,-)$.

We will use below the following elementary construction of Lawvere theories. Let $A$ be an object in a category $\mathcal{C}$ with finite products. Choose, for each $n \in \mathbb{N}$, a power $A^{n} \stackrel{\pi_{i}}{\longrightarrow} A$ such that $A^{n} \neq A^{m}$ for $n \neq m$. Then the full subcategory of $\mathcal{C}$ spanned by these powers is a Lawvere theory, which we will denote by $\mathcal{T}_{\mathcal{C}}[A]$. Note that in this notation the theory $\mathcal{T}_{\mathcal{V}}$ of a variety $\mathcal{V}$ as described in II. (a) above is $\mathcal{T}_{\mathcal{V} \text { op }}\left[F_{1}\right]$.

We note moreover that, given an object $A$ in a category $\mathcal{C}$ with coproducts, there is the monad $\mathbb{A}$ given by the adjunction $-\cdot A \dashv \mathcal{C}(A,-)$. Though this monad formally is related to the theory $T_{\mathcal{C}^{\text {op }}}[A]$ as $\mathbb{T}$ is related to $\mathcal{T}_{\mathcal{V}^{\text {op }}}\left[F_{\mathcal{V}} 1\right], S e t^{\mathbb{A}}$ will not be concretely equivalent to a variety in general.

\subsubsection{Characterizing varieties as concrete categories}

The characterization of varieties as concrete categories, that is, as categories $\mathcal{A}$ equipped with faithful functor $\mathcal{A} \stackrel{U}{\rightarrow}$ Set is essentially due to Linton 21. A concrete category $(\mathcal{V}, U)$ is concretely equivalent to a variety if only if (0) $\mathcal{V}$ has coequalizers, (1) $U$ has a left adjoint, (2) $U$ preserves and reflects regular epimorphisms, (3) $U$ preserves and reflects kernel pairs, and (4) $U$ preserves directed colimits. Assuming that $\mathcal{A}$ even is cocomplete and has kernel pairs and regular factorizations of morphisms $U$ is a representable functor $U \simeq \mathcal{A}(G,-)$ and one can replace the above conditions by the following ones: $G$ is (a) a regular generator, (b) regular projective (i.e., projective with respect to regular epimorphisms), and (c) finitely presentable (see e.g. 10, Thm 3.9.1] or [25]). We will call such $G$ a varietal generator.

One so obtains that for any varietal generator $G$ in a cocomplete category $\mathcal{A}$ with kernel pairs and regular factorizations of morphisms the variety $\mathcal{V}$ with $\mathcal{T}_{\mathcal{V}}=\left(\mathcal{T}_{\mathcal{A}^{\text {op }}}[G]\right)^{\text {op }}$ admits an equivalence $\mathcal{A} \stackrel{R_{G}}{\longrightarrow} \mathcal{V}$ with $|-| \circ R_{G}=\mathcal{A}(G,-)$.

\subsubsection{A universal property of varieties}

Maybe somewhat lesser known than what we recalled in the previous section is the characterization of varieties $\operatorname{Alg} \mathcal{T}$ as free completion of the category $\mathcal{T}^{\text {op }}$ under sifted colimits (see [3]). This is in detail: For every functor $F: \mathcal{T}^{\mathrm{op}} \rightarrow \mathcal{C}$ into a category $\mathcal{C}$ with sifted colimits there exists 
an essentially unique sifted colimit preserving functor $S_{F}: \operatorname{Alg} \mathcal{T} \rightarrow \mathcal{C}$ extending $F$ such that the assignment $F \mapsto S_{F}$ defines an equivalence between the categories $\left[\mathcal{T}^{\mathrm{op}}, \mathcal{C}\right]$ and sift $[\operatorname{Alg} \mathcal{T}, \mathcal{C}]$, the category of sifted colimit preserving functors from $\operatorname{Alg} \mathcal{T}$ to $\mathcal{C}$ (see [3, 4]).

Since this result will be of importance below we use the opportunity to describe the functor $S_{F}$ as the left Kan extension $\operatorname{Lan}_{Y}(F)$ as follows, where $Y$ denotes the embedding $\mathcal{T}$ op $\hookrightarrow \operatorname{Alg} \mathcal{T}$.

Proposition 2 For every functor $F: \mathcal{T}^{\mathrm{op}} \rightarrow \mathcal{C}$ into a category $\mathcal{C}$ with sifted colimits Lan $_{Y} F$ exists. If $\mathcal{C}$ in addition has finite coproducts, that is, if $\mathcal{C}$ is cocomplete the following hold.

1. $\operatorname{Lan}_{Y} F$ coincides with the restriction of the Yoneda extension of $F$.

2. Lan $_{Y} F$ coincides with the unique sifted colimit preserving extension $S_{F}$ of $F$.

3. $S_{F}=\operatorname{Lan}_{Y} F$ has a right adjoint $R_{F}$, if $F$ preserves finite coproducts.

The proof is based on the following lemma.

Lemma 3 ([4]) Alg $\mathcal{T}$ is closed in $S e t^{\mathcal{T}}$ under sifted colimits. For every $\mathcal{T}$-algebra $\mathcal{T} \stackrel{A}{\rightarrow}$ Set the category $\mathrm{El} A$ of elements of the functor $\mathrm{A}$ is sifted and coincides with the comma category $Y \downarrow A$.

Proof (of Proposition 2) The first statement and item 1 are clear by the construction of Kan extensions. By the lemma above the embedding Alg $\mathcal{T} \stackrel{I}{\rightarrow} S e t^{\mathcal{T}}$ preserves sifted colimits. Since the Yoneda extension is a left adjoint, $\operatorname{Lan}_{Y} F$ preserves these by item 1 and, hence, coincides with $S_{F}$. Concerning item 3 observe first, that the functor $\mathcal{T} \stackrel{\hat{R}_{F}(C)}{\longrightarrow}$ Set preserves finite products if $F^{\text {op }}$ does; hence, in this case $\hat{R}_{F}$ factors as $\mathcal{C} \stackrel{R_{F}}{\longrightarrow} \operatorname{Alg} \mathcal{T} \stackrel{I}{\rightarrow} S e t^{\mathcal{T}}$. By Equation (3) there are natural isomorphisms $\mathcal{C}\left(\operatorname{Lan}_{Y} F(A), C\right) \simeq \operatorname{Set}^{\mathcal{T}}\left(I(A), \hat{R}_{F} C\right) \simeq \operatorname{Alg} \mathcal{T}\left(A, R_{F} C\right)$; in other words there is an adjunction $\operatorname{Lan}_{Y} F \dashv R_{F}$.

The following diagram illustrates this situation. (Note, that the Yoneda extension $\operatorname{Lan}_{Y_{\text {Top }}} F$ will only exist if $\mathcal{C}$ in addition has finite coproducts, that is, if $\mathcal{C}$ is cocomplete.) We only include it in the diagram to indicate that in this case the adjunction $\operatorname{Lan}_{Y} F \dashv R_{F}$ appears as a restriction of the adjunction of the Yoneda extension).

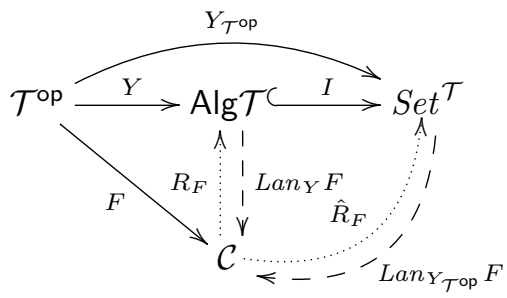

\subsubsection{Internal algebras in a category}

Given an arbitrary category $\mathcal{C}$ with finite products one defines categories $\operatorname{Alg}(\mathcal{T}, \mathcal{C}), \operatorname{Alg}(\Sigma, \mathcal{C})$ and $\operatorname{Alg}((\Sigma, \mathfrak{E}), \mathcal{C})$ of internal algebras in $\mathcal{C}$, equipped with underlying functors $|-|_{\mathcal{C}}$, by replacing in the definitions of $\operatorname{Alg} \Sigma, \operatorname{Alg}(\Sigma, \mathfrak{E})$ and $\operatorname{Alg} \mathcal{T}$, respectively, "set" by "C -object" and "map" by "C-C-morphism". For a variety $\mathcal{V}=\operatorname{Alg}(\Sigma, \mathfrak{E})$ one calls $\operatorname{Alg}((\Sigma, \mathfrak{E}), \mathcal{C})$ the category of internal $\mathcal{V}$-algebras in $\mathcal{C}$. A $\mathcal{V}$-algebra in $\mathcal{C}$, thus, is a pair $\left(A,\left(\sigma^{A}\right)_{\sigma \in \Sigma}\right)$ such that for each $(s, t) \in \mathfrak{E}$ Diagram (4), considered as a diagram in $\mathcal{C}$, commutes. Internal $\mathcal{V}$-algebras in $\mathcal{C}$ can alternatively be described as follows ([22, Chapter III.6],[16]): 
Fact 4 A $\mathcal{C}$-object $A$ is (the underlying object) of an internal $\mathcal{V}$-algebra iff, for each $\mathcal{C}$-object $C$, the set $\mathcal{C}(C, A)$ is (the underlying set) of a $\mathcal{V}$-algebra.

This is equivalent to saying that $\mathcal{V}$-algebras $\mathrm{A}$ in $\mathcal{C}$ with underlying object $A$ correspond essentially one-to-one to functors $R_{\mathrm{A}}$ making the following diagram commute.

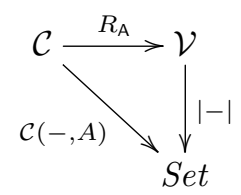

The following facts either follow by direct generalization or are obvious.

Fact 5 If $\mathcal{T}_{\mathcal{V}}$ is the Lawvere theory of a variety $\mathcal{V}=\operatorname{Alg}(\Sigma, \mathfrak{E})$, then the following hold.

1. The functor $\operatorname{Alg}\left(\mathcal{T}_{\mathcal{V}}, \mathcal{C}\right) \stackrel{|-|_{\mathcal{C}}}{\longrightarrow} \mathcal{C}$ creates limits and reflects isomorphisms.

For every $t \in \mathcal{T}_{\Sigma}(n, 1)$ there is a natural transformation $\tilde{t}:|-|_{\mathcal{C}}^{n} \Rightarrow|-|_{\mathcal{C}}$, whose components are the $\mathcal{C}$-morphisms $\mathrm{A}(t):|\mathrm{A}|_{\mathcal{C}}^{n} \rightarrow|\mathrm{A}|_{\mathcal{C}}$.

2. $\operatorname{Alg}\left(\mathcal{T}_{\mathcal{V}}, \mathcal{C}\right)$ is concretely equivalent over $\mathcal{C}$ to $\operatorname{Alg}((\Sigma, \mathfrak{E}), \mathcal{C})$, the full subcategory $\operatorname{Equ}(\tilde{s}, \tilde{t})_{s=t \in \mathfrak{E}}$ of the category $\operatorname{Alg}(\Sigma, \mathcal{C})$.

3. Morphisms $\mathcal{S} \stackrel{\Phi}{\rightarrow} \mathcal{T}$ of Lawvere theories determine functors $\mathcal{C} \Phi^{*}: \operatorname{Alg}(\mathcal{T}, \mathcal{C}) \rightarrow \operatorname{Alg}(\mathcal{S}, \mathcal{C})$ given by $(\mathcal{T} \stackrel{\mathrm{A}}{\rightarrow} \mathcal{C}) \mapsto(\mathcal{S} \stackrel{\Phi}{\rightarrow} \mathcal{T} \stackrel{\mathrm{A}}{\rightarrow} \mathcal{C})$.

4. Every product preserving functor $\mathcal{C} \stackrel{S}{\rightarrow} \mathcal{D}$ between categories with finite products maps $\mathcal{T}$-algebras to $\mathcal{T}$-algebras, that is, $S$ can be lifted to a functor $\operatorname{Alg}(\mathcal{T}, \mathcal{C}) \stackrel{{ }^{\top} S}{\longrightarrow} \operatorname{Alg}(\mathcal{T}, \mathcal{D})$ by mapping a $\mathcal{T}$-algebra $\mathrm{A}$ to $S \circ \mathrm{A} .{ }^{\mathcal{T}} S$ preserves all limits which are preserved by $S$. In the language of universal algebra, one has a functor $\operatorname{Alg}(\mathcal{V}, \mathcal{C}) \stackrel{\nu_{S}}{\longrightarrow} \operatorname{Alg}(\mathcal{V}, \mathcal{D})$ such that $\mathcal{\nu}_{S}(\mathrm{~A})=\left(S(A),\left(S\left(\sigma^{\mathrm{A}}\right)\right)_{\sigma \in \Sigma}\right)$.

However, unlike to the case of $\mathcal{C}=\operatorname{Set}$, neither the forgetful functor $\operatorname{Alg}(\mathcal{T}, \mathcal{C}) \stackrel{|-|_{\mathcal{C}}}{\longrightarrow} \mathcal{C}$ nor the functors $\mathcal{C} \Phi^{*}$ have left adjoints in general. They do so, if $\mathcal{C}$ is a locally finitely presentable category, and in this case $\operatorname{Alg}(\mathcal{T}, \mathcal{C})$ is locally finitely presentable (see e.g. [27]).

\section{Internal coalgebras}

\subsection{Descriptions of the category of coalgebras}

If $\mathcal{C}$ is a category with finite coproducts one calls an internal algebra in $\mathcal{C}^{\mathrm{op}}$ an internal coalgebra in $\mathcal{C}$. This generalizes the terminology used already by Kan 19] as early as 1958 in the special situation, where $\mathcal{C}=$ Mon and $\mathcal{V}=G r p$. In this section we describe various equivalent descriptions of this concept in some detail. 


\subsubsection{Coalgebras for a variety}

Definition 6 Let $\mathcal{C}$ be a category with finite coproducts.

For any Lawvere theory $\mathcal{T}$ the category of internal $\mathcal{T}$-coalgebras in $\mathcal{C}$ is the category Coalg $(\mathcal{T}, \mathcal{C})=\operatorname{Alg}\left(\mathcal{T}, \mathcal{C}^{\text {op }}\right)^{\text {op }}$.

For any variety $\mathcal{V}=\operatorname{Alg}(\Sigma, \mathfrak{E})$ the category internal $\mathcal{V}$-coalgebras in $\mathcal{C}$ (or internal $(\Sigma, \mathfrak{E}$ )coalgebras $)$ is the category $\operatorname{Coalg}(\mathcal{V}, \mathcal{C})=\operatorname{Alg}\left(\mathcal{V}, \mathcal{C}^{\text {op }}\right)^{\text {op }}$.

Facts 7 1. Coalg $(\mathcal{T}, \mathcal{C})$ is the category of finite coproduct preserving functors $\mathcal{T}^{\text {op }} \rightarrow \mathcal{C}$.

2. $\operatorname{Coa} \lg (\mathcal{V}, \mathcal{C})=\operatorname{Coalg}((\Sigma, \mathfrak{E}), \mathcal{C})$ is, by dualization of Facts 52 , the joint equifier $\operatorname{Equ}(\hat{s}, \hat{t})_{s=t \in \mathfrak{E}}$ in Coalg $(\Sigma, \mathcal{C})$, where $\hat{t}:=\tilde{t}^{\mathrm{op}}:\|-\| \Rightarrow n \cdot\|-\|$ is the natural transformation determined by $t \in \mathcal{T}_{\Sigma}(n, 1) . t_{\mathrm{A}}:=\hat{t}_{\mathrm{A}}: A \rightarrow n \cdot A$ is the interpretation of $t$ in an internal $\Sigma$-algebra in $\mathcal{C}^{\circ \text { op }}$, for each such $t$. Moreover, $\operatorname{Coalg}(\mathcal{V}, \mathcal{C}) \simeq \operatorname{Coalg}\left(\mathcal{T}_{\mathcal{V}}, \mathcal{C}\right)$.

Adopting the language of universal algebra, a $\Sigma$-coalgebra in $\mathcal{C}$ is a pair $\mathrm{A}=\left(A,\left(A \stackrel{\sigma_{\mathrm{A}}}{\longrightarrow}\right.\right.$ $\left.n \cdot A)_{\sigma \in \Sigma_{n}}\right)$, where $A$ is a $\mathcal{C}$-object and $A \stackrel{\sigma_{\mathrm{A}}}{\longrightarrow} n \cdot A$ is a $\mathcal{C}$-morphism, called the $n$-ary co-operation determined by $\sigma \in \Sigma_{n}$. A $(\Sigma, \mathfrak{E})$-coalgebra in $\mathcal{C}$ then is a $\Sigma$-coalgebra in $\mathcal{C}$ making all Diagrams (4) in $\mathcal{C}^{\mathrm{op}}$ commute. This is often expressed by saying that it satisfies duals of the equational axioms defining $\mathcal{V}$, which means explicitly that each of the following diagrams commutes in $\mathcal{C}$.

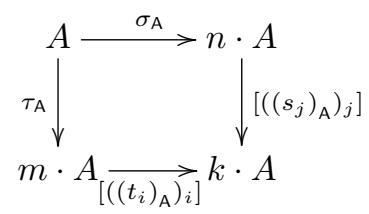

The following diagrams from [19], with $C \stackrel{\mu}{\rightarrow} C+C$ the comultiplication, $C \stackrel{\epsilon}{\rightarrow} 0$ the counit, and $C \stackrel{\iota}{\rightarrow}$ the coinversion, displaying the axioms of a cogroup may serve as an illustration.

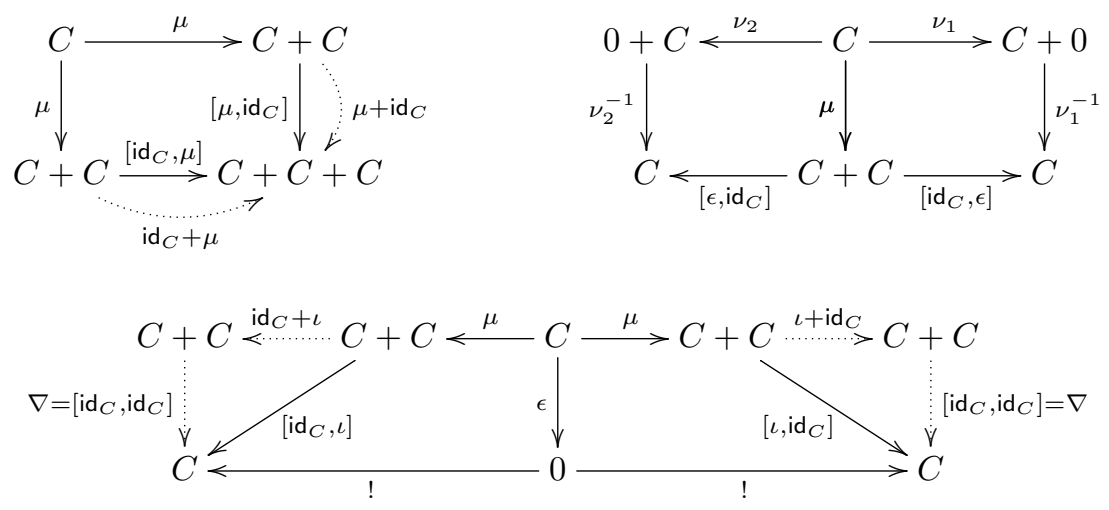

Remark 8 It may happen that $\operatorname{Coalg}(\mathcal{V}, \mathcal{C})$ is the category 1 . This is the case for example if $\mathcal{C}(C, 0)=\emptyset$ for all $C \neq 0$ (as in $S e t)$ and $\mathcal{V}$ has no constants. An example is Coalg $(M o n, S e t)$.

It also may happen that $\operatorname{Coalg}(\mathcal{V}, \mathcal{C})$ is isomorphic to the category $\mathcal{C}$. Examples are the categories Coalg $($ Mon, $\mathcal{C})$ and $\operatorname{Coalg}\left({ }_{c}\right.$ Mon, $\left.\mathcal{C}\right)$, if $\mathcal{C}$ is a semi-additive category. This is due to the familiar fact that the (only) comonoids in a cartesian monoidal category $\mathcal{C}$ are the triples 
$\left(C, \Delta_{C}, !_{C}\right)$ with $C \stackrel{\Delta_{C}}{\longrightarrow} C \times C$ the diagonal and $C \stackrel{!_{C}}{\longrightarrow} 1$ the unique morphism, and that these are cocommutative.

Other examples are Coalg $(G r p, \mathcal{C})$ and $\operatorname{Coalg}(A b, \mathcal{C})$ if $\mathcal{C}$ is additive. In this case, for each $\mathcal{C}$-object $C$, the hom-functor $\mathcal{C}(C,-)$ can be lifted to a functor $\mathcal{C} \rightarrow A b$. In other words, $C$ carries the structure of an $A b$-coalgebra in $\mathcal{C}$. By the above the claim follows and one obtains equivalences of categories Coalg $(G r p, \mathcal{C}) \simeq \mathcal{C} \simeq \operatorname{Coalg}(A b, \mathcal{C})$.

\subsubsection{Coalgebras as representable functors}

Definition 9 Given a Lawvere theory $\mathcal{T}$ and $\mathcal{C}$ a category with finite coproducts. A functor $\mathcal{C} \stackrel{R_{A}}{\longrightarrow} \operatorname{Alg} \mathcal{T}$ is called $\mathcal{T}$-representable (on $\mathcal{C}$ ) by $A \in$ obC or an $\operatorname{Alg} \mathcal{T}$-lift of $\mathcal{C}(A,-$ ), if $\mathcal{C} \rightarrow$ Alg $\mathcal{T} \stackrel{|-|}{\longrightarrow} S e t=\mathcal{C} \stackrel{\mathcal{C}(A,-)}{\longrightarrow}$ Set for some $\mathcal{C}$-object $A$, that is, if diagram (6) commutes.

$\operatorname{Rep}(\mathcal{T}, \mathcal{C})$ denotes the category of $\mathcal{T}$-representable functors on $\mathcal{C}$ with all natural transformations as its morphisms.

Occasionally we will rather talk about $\mathcal{V}$-lifts and $\mathcal{V}$-representable functors, if $\mathcal{T}=\mathcal{T}_{\mathcal{V}}$ for a variety $\mathcal{V}$.

Remarks 10 1. For any morphism $R_{A} \stackrel{\mu}{\Rightarrow} R_{A^{\prime}}$ in $\operatorname{Rep}(\mathcal{T}, \mathcal{C})$ the natural transformation $\mathcal{C}(A,-) \stackrel{|\mu|}{\Rightarrow} \mathcal{C}\left(A^{\prime},-\right)$ determines by the Yoneda Lemma a $\mathcal{C}$-morphism $A^{\prime} \stackrel{f_{\mu}}{\longrightarrow} A$ between the representing objects. $\operatorname{Rep}(\mathcal{T}, \mathcal{C})^{\text {op }}$ then is concrete over $\mathcal{C}$ by means of the faithful functor $\{-\}_{\mathcal{T}}$ given by $\left(R \stackrel{\mu}{\rightarrow} R^{\prime}\right) \mapsto\left(A^{\prime} \stackrel{f_{\mu}}{\longrightarrow} A\right)$.

2. For any $\mathcal{V}$-representable functor $R_{A}$ one has for each set $X$ the natural isomorphism

$$
\mathcal{V}\left(F X, R_{A^{-}}\right) \simeq \mathcal{V}\left(F 1, R_{A}-\right)^{X} \simeq \mathcal{C}(A,-)^{X} \simeq \mathcal{C}(X \cdot A,-) .
$$

Identifying $\mathcal{V}\left(F X, R_{A}-\right)$ and $\mathcal{C}(X \cdot A,-)$, for each $\mathcal{V}$-homomorphism $F 1 \stackrel{g}{\rightarrow} F X$ the natural transformation $\mathcal{V}\left(g, R_{A^{-}}\right): \mathcal{V}\left(F X, R_{A}-\right) \Rightarrow \mathcal{V}\left(F 1, R_{A^{-}}\right)$is a natural transformation $\mathcal{C}(X \cdot A,-) \Rightarrow \mathcal{C}(A,-)$ and so determines by the Yoneda lemma a $\mathcal{C}$-morphism $X \cdot A \stackrel{\gamma}{\rightarrow} A$.

Dualizing Fact 4 one obtains

Lemma 11 For every Lawvere theory $\mathcal{T}$ and every category $\mathcal{C}$ with finite coproducts the assignment $\mathrm{A} \mapsto R_{\mathrm{A}}$ defines an equivalence, concrete over $\mathcal{C}$,

$$
\operatorname{Coalg}(\mathcal{T}, \mathcal{C}) \simeq \operatorname{Rep}(\mathcal{T}, \mathcal{C})^{\text {op }} .
$$

In more detail: If $\mathcal{T}^{\text {op }} \stackrel{\mathrm{A}}{\rightarrow} \mathcal{C}$ is a coalgebra with $\mathrm{A}\left(T_{1}\right)=A$, then the functor $\mathcal{C}(A,-)$ has the $\mathcal{T}$-lift $\mathcal{C} \stackrel{R_{\mathrm{A}}}{\longrightarrow}$ Alg $\mathcal{T}$ given by the assignment $C \mapsto \mathcal{T} \stackrel{\mathrm{A}^{\mathrm{op}}}{\longrightarrow} \mathcal{C}^{\text {op }} \stackrel{\mathcal{C}(-, C)}{\longrightarrow}$ Set. Conversely, for any $\mathcal{T}$-representable functor $R_{A}$ one obtains a coalgebra $\mathcal{T}^{\text {op }} \stackrel{\mathrm{A}}{\rightarrow} \mathcal{C}$ with $\mathrm{A} T_{1}=A$ by the assignment $F 1 \stackrel{g}{\rightarrow} F n \mapsto A \stackrel{\gamma}{\rightarrow} n \cdot A$ defined in item 2. above. For a more conceptual description of this correspondence see below.

Remark 12 A representable functor $\mathcal{C}(A,-)$ may have non-isomorphic $\operatorname{Alg} \mathcal{T}$-lifts $H, H^{\prime}: \mathcal{C} \rightarrow$ Alg $\mathcal{T}$, equivalently, a $\mathcal{C}$-object may carry more than one structure of a $\mathcal{T}$-coalgebra.

For example there are the functors $\Phi, \Psi: \operatorname{Ring} \rightarrow$ Mon mapping a unital ring $R$ to its additive monoid $(R,+, 0)$ and its multiplicative monoid $(R, \times, 1)$, respectively. These are Mon-lifts of the representable functor $\operatorname{Ring}(\mathbb{Z}[x],-)$, which is up to equivalence the forgetful functor of Ring. See Theorem 20 for a complete discussion of this phenomenon. 


\subsubsection{Coalgebras as adjunctions}

For every adjunction $L \dashv R$ : $\operatorname{Alg} \mathcal{T} \rightarrow \mathcal{C}$, where $\mathcal{C}$ is a category with finite coproducts, the right adjoint $R$ is $\mathcal{T}$-representable. In fact, the natural isomorphism $\mathcal{C}(L F 1,-) \simeq \operatorname{Alg} \mathcal{T}(F 1, R-)$ is equivalent to $|-| \circ R \simeq \mathcal{C}(L F 1,-)$. The converse holds, if $\mathcal{C}$ is cocomplete. This has been claimed already, for the case of $\mathcal{C}$ being cocomplete, by Freyd (without giving a proof) and is an immediate consequence of Beck's lifting theorem for adjunctions along a monadic functor (see e.g. 10]). For the sake of the reader interested in an algebraically flavoured construction of this left adjoint we explain below how this argument works in the given situation, correcting at the same time the proof given in $[8]$.

The resulting correspondences between coalgebras, representable functors and adjunctions can be seen more conceptually as follows, where $\operatorname{Adj}(\mathcal{D}, \mathcal{C})$ denotes the category of adjunctions from $\mathcal{D}$ to $\mathcal{C}$ as defined in 22$]$ :

Theorem 13 For every Lawvere theory $\mathcal{T}$ and every cocomplete category $\mathcal{C}$ there are equivalences

$$
\operatorname{Coalg}(\mathcal{T}, \mathcal{C}) \simeq \operatorname{Rep}(\mathcal{T}, \mathcal{C})^{\mathrm{op}} \simeq \operatorname{Adj}(\operatorname{Alg} \mathcal{T}, \mathcal{C})
$$

In more detail:

1. The left adjoint corresponding to a $\mathcal{T}$-coalgebra $\mathrm{A}$ in $\mathcal{C}$ is its left Kan extension $\operatorname{Lan}_{Y} \mathrm{~A}$. It is the sifted colimit preserving extension of $\mathrm{A}$ as well.

2. The $\mathcal{T}$-representable functor corresponding to a $T$-coalgebra $\mathrm{A}$ in $\mathcal{C}$ is the right adjoint of $\operatorname{Lan}_{Y} \mathrm{~A}$

3. The T-coalgebra $\mathrm{A}$ in $\mathcal{C}$ corresponding to a left adjoint functor $\mathrm{Alg} \mathcal{T} \stackrel{L}{\rightarrow} \mathcal{C}$ is its restriction to $\mathcal{T}^{\mathrm{op}}$, considered as a subcategory of $\mathrm{Alg} \mathcal{T}$.

Indeed, by Proposition 2 there is the adjunction $L_{\mathrm{A}}=\operatorname{Lan}_{Y} \mathrm{~A} \dashv R_{\mathrm{A}}$ for every $\mathcal{T}$-coalgebra $\mathrm{A}$ in $\mathcal{C}$. By its definition the functor $R_{\mathrm{A}}$ is the $\mathcal{T}$-representable functor represented by $\mathrm{A}\left(T_{1}\right)$ (see Section 2.1.2. Noting further that the equation $S_{F}=\operatorname{Lan}_{Y} F$ implies that the correspondence of item 3 is an equivalence by the definition of the free cocompletion (see Section 1.2.4), this theorem only rephrases Proposition 2.

Remarks 14 1. $\operatorname{Rep}(\mathcal{T}, \mathcal{C})$ is equivalent to the category $\operatorname{Radj}(\mathcal{C}, \operatorname{Alg} \mathcal{T})$ of right adjoints from $\mathcal{C}$ to $\operatorname{Alg} \mathcal{T}$ with natural transformations as morphisms, while $\operatorname{Adj}(\operatorname{Alg} \mathcal{T}, \mathcal{C})$ is equivalent to the category $\operatorname{Ladj}(\operatorname{Alg} \mathcal{T}, \mathcal{C})$ of left adjoints (equivalently, by the Special Adjoint Functor Theorem, to ${ }_{\operatorname{coc}}[\operatorname{Alg} \mathcal{T}, \mathcal{C}]$, the category of cocontinous functors) from $\operatorname{Alg} \mathcal{T}$ to $\mathcal{C}$ with natural transformations as morphisms. $\operatorname{Adj}(\operatorname{Alg} \mathcal{T}, \mathcal{C})$ becomes concrete over $\mathcal{C}$ by assigning to a morphism $L \dashv R \stackrel{\langle\sigma, \rho\rangle}{\longrightarrow} L^{\prime} \dashv R^{\prime}$, that is, a pair of conjugate transformations, the $\mathcal{C}$-morphism $L(F 1) \stackrel{\sigma_{F 1}}{\longrightarrow} L^{\prime}(F 1)$, the $\sigma$-component at the free $\mathcal{T}$-algebra over a singleton.

2. It is easy to see that for any natural transformation $L \stackrel{\sigma}{\Rightarrow} L^{\prime}$ the underlying $\mathcal{C}$-morphism of the right adjoint $R$ of $L$ is $\{\rho\}_{\mathcal{T}}$, where $\langle\sigma, \rho\rangle$ is a conjugate pair. Thus, the equivalences of the theorem are concrete over $\mathcal{C}$.

3. Denoting by $\mathcal{V}$ the variety corresponding to $\mathcal{T}$, the $\mathcal{V}$-coalgebra $\mathrm{A}$ in $\mathcal{C}$ corresponding to an adjunction $L \dashv R: \mathcal{V} \rightarrow \mathcal{C}$ then is, in the language of universal algebra, $\mathrm{A}=$ $\left(L\left(F_{\mathcal{V}} 1\right),\left(\sigma_{\mathrm{A}}\right)_{\sigma \in \Sigma}\right)$, with co-operations $\sigma_{\mathrm{A}}=L\left(F_{\mathcal{V}} 1\right) \stackrel{L(\bar{\sigma})}{\longrightarrow} L\left(F_{\mathcal{V}} n\right), \sigma \in \Sigma_{n}$ (see Item III. of Section 1.2.2). 


\section{The Eilenberg-Watts Theorem.}

The theorem above can be seen as the utmost generalization of the Eilenberg-Watts Theorem, since combining it with Facts 7 we the get the equivalence

$$
\operatorname{Coalg}\left({ }_{R} M o d,{ }_{S} M o d\right) \simeq \operatorname{Ladj}\left({ }_{R} M o d,{ }_{S} M o d\right) .
$$

It thus suffices to show that Coalg $\left({ }_{R} \operatorname{Mod},{ }_{S} M o d\right)$ is nothing but the category ${ }_{S} M o d_{R}$ of left $S$-, right $R$-bimodules. We prove something more in order to show how the respective result from 23 fits into this picture. Let $\mathcal{C}$ be a cocomplete and additive category. Then, for every $\mathcal{C}$ object $C$ the set $\mathcal{C}(C, C)$ carries the structure of a ring $\operatorname{End}_{\mathcal{C}}(C)$ and the categories $\operatorname{Coalg}(A b, \mathcal{C})$ and $A b$ are isomorphic (see Section 2.1.1); hence, Coalg $\left({ }_{R} \operatorname{Mod}, \mathcal{C}\right)$ is isomorphic to the category of left $R$-objects in $\mathcal{C}$ in the sense of [24], that is, of pairs $(C, \rho)$, where $R \stackrel{\rho}{\rightarrow} \operatorname{End}_{\mathcal{C}}(C)$ is a ring homomorphism. In particular the category Coalg $\left({ }_{R} \operatorname{Mod},{ }_{S} M o d\right)$ is nothing but the category ${ }_{S} \operatorname{Mod}_{R}$ of left $S$-, right $R$-bimodules. Note that this argument strengthens the original result in that the assumption of additivity is not needed. (In fact, it wasn't needed from the very beginning since every finite coproduct preserving functor between additive categories is additive.).

\section{An algebraic proof of right adjointness of $\mathcal{V}$-representable functors.}

Choose, for any algebra $A$ in a variety $\mathcal{V}$, its canonical presentation by generators and relations given by the coequalizer diagram $F\left|K_{A}\right| \stackrel{r_{A}}{\underset{s_{A}}{\longrightarrow}} F|A| \stackrel{\epsilon_{A}}{\longrightarrow} A$, where $\epsilon_{A}$ is the counit of the adjunction $F \dashv|-|, \quad K_{A} \underset{q}{\stackrel{p}{\longrightarrow}} F|A| \stackrel{\epsilon_{A}}{\longrightarrow} A$ its congruence relation (kernel pair) and $\left(r_{A}, s_{A}\right)$ is the pair of homomorphic extensions of the projections $p$ and $q$. Denote by $\rho_{A}$ and $\sigma_{A}$ the $\mathcal{C}$ morphisms $|A| \cdot R \rightarrow\left|K_{A}\right| \cdot R$ corresponding to $r_{A}$ and $s_{A}$ according to Remarks 102 . and let $|A| \cdot R \underset{\sigma_{A}}{\stackrel{\rho_{A}}{\longrightarrow}}\left|K_{A}\right| \cdot R \stackrel{v_{A}}{\longrightarrow} L A$ be a coequalizer diagram. Since contravariant hom-functors map coequalizers to equalizers one obtains, for each $C$ in $\mathcal{C}$, the following equalizer diagrams in Set.

$$
\begin{array}{r}
\mathcal{V}(A, H C) \stackrel{\mathcal{V}\left(\epsilon_{A}, H C\right)}{\longrightarrow} \mathcal{C}(|A| \cdot R, C) \underset{\mathcal{C}\left(\sigma_{A}, C\right)}{\stackrel{\mathcal{C}\left(\rho_{A}, C\right)}{\longrightarrow}} \mathcal{C}\left(\left|K_{A}\right| \cdot R, C\right) \\
\mathcal{C}(L A, C) \stackrel{\mathcal{C}\left(v_{A}, C\right)}{\longrightarrow} \mathcal{C}(|A| \cdot R, C) \underset{\mathcal{C}\left(\rho_{A}, C\right)}{\stackrel{\mathcal{C}\left(\sigma_{A}, C\right)}{\longrightarrow}} \mathcal{C}\left(\left|K_{A}\right| \cdot R, C\right)
\end{array}
$$

Hence, for each $C$ in $\mathcal{C}$ and each $A$ in $\mathcal{V}$, there is a bijection $\mathcal{C}(L A, C) \stackrel{\alpha_{A, C}}{\longrightarrow} \mathcal{V}(A, H C)$

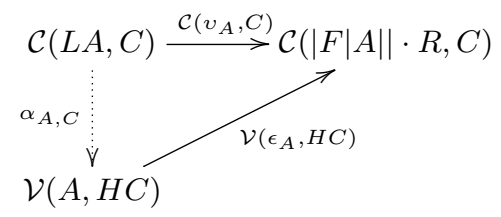

The claim now follows by [22, Chapter IV.1, Corollary 2], provided that these bijections are natural in $C$. But this is obvious: Since $\mathcal{V}\left(\epsilon_{A}, H-\right)$ and $\mathcal{C}\left(v_{A},-\right)$ are natural and $\mathcal{V}\left(\epsilon_{A}, H C\right)$ is monic, for each $C$ in $\mathcal{C}$, commutativity of Diagram (8) implies that the family $\left(\alpha_{A, C}\right)_{C}$ is natural. It is this naturality condition of which in the proof in [8] is not (and cannot be) taken care of, due to the fact that there is only chosen an arbitrary presentation by generators and relations of the algebra $A$ at the beginning. 


\subsubsection{Coalgebras for monad}

Recall that a $\mathcal{C}$-object $A$ carries the structure of a $\mathcal{T}$-coalgebra in $\mathcal{C}$ iff there exists a morphism of theories $\mathcal{T} \stackrel{\phi}{\rightarrow} \mathcal{T}_{\mathcal{C}^{\text {op }}}[A]$. If $\mathcal{C}$ is cocomplete, such $\phi$ extends to a monad morphism from the free algebra monad $\mathbb{T}$ of $A \lg \mathcal{T}$ to the monad $\mathbb{A}$ given by the adjunction $-\cdot A \dashv \mathcal{C}(A,-)$.

Since monad morphisms $\mathbb{T} \stackrel{\phi}{\rightarrow} \mathbb{A}$ correspond bijectively to functors $S e t^{\mathbb{A}} \stackrel{\phi^{*}}{\longrightarrow} S e t^{\mathbb{T}}$ commuting with the forgetful functors (see e.g. [11, Section 3.6]) we obtain a bijective correspondence between such pairs $\mathrm{A}=(A, \phi)$ and $\mathbb{T}$-representable functors $\mathcal{C} \stackrel{R_{\mathrm{A}}}{\longrightarrow}$ Set $t^{\mathbb{T}}$, that is, functors $R_{\mathrm{A}}$ such that $U_{\mathbb{T}} \circ R_{\mathrm{A}}=\mathcal{C}(A, C)$, for each $C$ in $\mathcal{C}$, and these are nothing but $\mathcal{T}$-representable functors, since $S e t^{\mathbb{T}} \simeq \operatorname{Alg} \mathcal{T}$.

Hence, a $\mathcal{C}$-object $A$ carries the structure of a $\mathcal{T}$-coalgebra $\mathrm{A}$ in $\mathcal{C}$ iff there exists a morphism of monads $\mathbb{T} \stackrel{\phi}{\rightarrow} \mathbb{A}$, and the $\mathcal{T}$-representable functor $R_{\mathrm{A}}$ corresponding to $\mathrm{A}$ is nothing but $\mathcal{C} \stackrel{K_{A}}{\longrightarrow}$ $S e t^{\mathbb{A}} \stackrel{\phi^{*}}{\longrightarrow} S e t^{\mathbb{T}} \simeq \operatorname{Alg} \mathcal{T}$ where $K_{A}$ is the comparison functor of the adjunction $-\cdot A \dashv \mathcal{C}(A,-)$. Thus, the following definition specializes for finitary monads to the case discussed so far and allows for an obvious generalization of Theorem 13 from finitary monads on Set to arbitrary ones.

Definition 15 Let $\mathcal{C}$ be a category with arbitrary coproducts and $\mathbb{T}$ be a monad on Set. A $\mathbb{T}$-coalgebra in $\mathcal{C}$ is a pair $\mathrm{A}=(A, \phi)$, where $A$ is $\mathcal{C}$-object and $\mathbb{T} \stackrel{\phi}{\rightarrow} \mathbb{A}$ a monad morphism.

A morphism $(A, \phi) \stackrel{f}{\rightarrow}(B, \psi)$ of $\mathbb{T}$-coalgebras is a $\mathcal{C}$-morphism $A \stackrel{f}{\rightarrow} B$ such that $\mathcal{C}(f,-)$ lifts to a natural transformation $R_{\mathrm{B}} \Rightarrow R_{\mathrm{A}}$, that is, if for each $C$ the map $\mathcal{C}(B, C) \stackrel{\mathcal{C}(f, C)}{\longrightarrow} \mathcal{C}(A, C)$ is a $\mathbb{T}$-morphism.

It is easy to see that some of the theoretic results below hold, if the assumption $\mathcal{T}$ is a Lawvere theory would be replaced by $\mathbb{T}$ is a monad on Set and, correspondingly $\operatorname{Alg} \mathcal{T}$ by $S e t^{\mathbb{T}}$. This applies in particular for Proposition 16, Theorem 20, and Proposition 22.

\subsection{Some examples, counter examples and applications}

Cogroups. For historical reasons only we start with the probably first example of cogroups in a category with finite coproducts $\mathcal{C}$, that is of $G r p$-coalgebras in $\mathcal{C}$, from homotopy theory (see [19, [16]). Let $\mathcal{C}=H T o p$ be the category of pointed topological space as objects and homotopy classes $\bar{f}$ of base-point preserving continuous maps $f$ as morphisms. The coproduct of two pointed space $\left(X, x_{0}\right)$ and $\left(Y, y_{0}\right)$ in $H T o p$ is their wedge sum $X \vee Y$, the topological sum of $X$ and $Y$ with $x_{0}$ and $y_{0}$ identified and taken as its base point.

By definition the $n^{\text {th }}$ homotopy group $\pi_{n}\left(X, x_{0}\right)$ of a pointed space $\left(X, x_{0}\right)$ has as its underlying set the hom-set HTop $\left(\left(S^{n}, s_{0}\right),\left(X, x_{0}\right)\right)$. Thus, the functor HTop $\stackrel{\pi_{n}}{\longrightarrow}$ Grp is a $G r p$-representable functor with representing object $S^{n}$. The corresponding cogroup structure is described as follows:

When collapsing an equator through $s_{0}$ of a pointed $n$-sphere $\left(S^{n}, s_{0}\right)$ to $s_{0}$, the quotient is $S^{n} \stackrel{\mu}{\rightarrow} S^{n} \vee S^{n}$. The multiplication of $\pi_{n}\left(X, x_{0}\right)$ is given by $\bar{f} \star \bar{g}=\overline{[f, g] \circ \mu}$, the unit is $1=\overline{x_{0}}$ with $x_{0}$ the constant map with value $x_{0}$, and the inversion is given by $\bar{f} \mapsto \overline{f \circ \iota}$ where $\iota$ is a change of orientation of $S^{n}$. Then proving that these data define a group is equivalent to proving that $\left(S^{n}, s_{0}\right)$ is a cogroup in HTop with comultiplication $\mu$ and inversion $\iota$. 
Modules. Let $\mathcal{V}$ be the variety $\operatorname{Mod}_{R}$ for a commutative ring and $M$ an $R$-module. The internal hom-functor $[M,-]: \operatorname{Mod}_{R} \rightarrow \operatorname{Mod}_{R}$ is $\mathcal{V}$-representable with representing object $M$ and has a left adjoint $M \otimes-$. The corresponding coalgebra has as its underlying object the module $M$ and the co-operations $M \stackrel{+}{\rightarrow} M+M$ and $M \stackrel{r_{M}}{\longrightarrow} M$ for each $r \in R$, which are by Theorem 13 the homomorphisms $M \simeq M \otimes R \stackrel{M \otimes+}{\longrightarrow} M \otimes R^{2} \simeq M^{2}$ and $M \simeq M \otimes R \stackrel{M \otimes r \cdot-}{\longrightarrow} M \otimes R \simeq M$, respectively. Thus, the coalgebra structure on $M$ is trivial in that it only "adds" the diagonal.

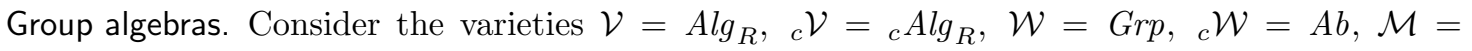
Mon, ${ }_{c} \mathcal{M}={ }_{c} M o n$ and $\mathcal{U}=\operatorname{Mod}_{R}$. The following diagram displays the (obvious) forgetful functors, where $\Phi^{*}(A)$ is the additive group of an algebra $A$, while $\Psi^{*}(A)$ is its multiplicative monoid, and the functor $(-)^{\times}$assigns to a (commutative) monoid its (abelian) group of invertible elements.

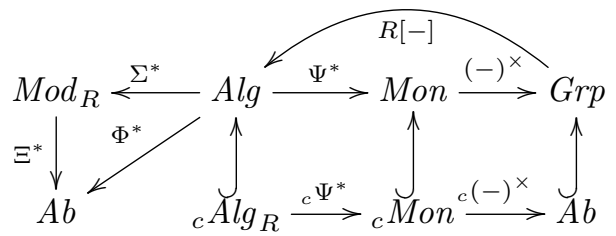

Being algebraic all forgetful functors have a left adjoint; the functors $(-)^{\times}$and ${ }_{c}(-)^{\times}$are right adjoints of the respective embeddings $\Lambda^{*}$ and ${ }_{c} \Lambda^{*}$. The left adjoint of $(-)^{\times} \circ \Psi^{*}$ is the group algebra functor $R[-]$ (note that $R[-]=\Psi_{*} \circ E$ where $E$ is the embedding $G r p \hookrightarrow M o n)$. The adjunction $R[-] \dashv(-)^{\times} \circ \Psi^{*}: G r p \rightarrow A l g_{R}$ hence determines a cogroup in $A l g_{R}$. In the sequel we will omit the subscript ${ }_{c}$ at functors if no confusion is possible.

Hopf structures. Since the monoidal structure of ${ }_{c} A l g_{R}$ coincides with the cocartesian structure,

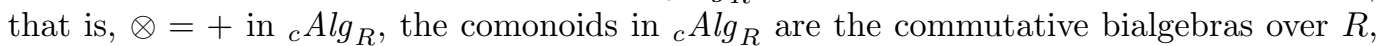
that is, Coalg $\left(\right.$ Mon, $\left.{ }_{c} A l g_{R}\right) \simeq{ }_{c} \operatorname{Bialg}_{R}$, and Coalg $\left({ }_{c}\right.$ Mon,$\left.{ }_{c} A l g_{R}\right) \simeq{ }_{b i}$ Bialg $_{R}$, the category of bi-commutative bialgebras. Similarly, the cogroups in ${ }_{c} A l g_{R}$ are the commutative Hopf

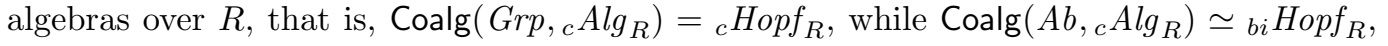
the category of bi-commutative Hopf algebras over $R$.

Denoting by $A b \stackrel{\Lambda^{*}}{\longrightarrow}{ }_{c}$ Mon the forgetful functor, the embedding ${ }_{b i} H_{o p f}{ }_{R} \hookrightarrow{ }_{b i}$ Bialg $_{R}$ is

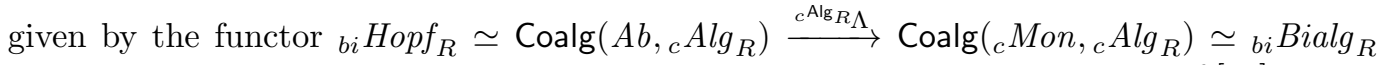
and, thus, has a right adjoint by Proposition 16 below. This improves a result of [26], where this fact has been shown for the case of absolutely flat rings only.

Obviously the adjunction $R[-] \dashv(-)^{\times} \circ \Psi^{*}$ of the previous example restricts to an adjunction ${ }^{A b} R[-] \dashv(-)^{\times} \circ \Psi^{*}: A b \rightarrow{ }_{c} A g_{R}$ and so determines a bicommutative Hopf algebra. The familiar Hopf algebra structure on a commutative group algebra $R[G]$ then is given by evaluation of the functor Coalg $(A b, A b) \stackrel{{ }^{A b} R[-]}{\longrightarrow} \operatorname{Coalg}\left(A b,{ }_{c} A l g, g_{R}\right) \simeq{ }_{b i} H o p f$ at the abelian group $G$, since Coalg $(A b, A b) \simeq A b$ by the Eckmann-Hilton argument.

A counter example. The full subcategory Tor of $A b$ spanned by all commutative torsion groups is a coreflective subcategory of (hence a comonadic category over) $A b$ but it is not of the form $\operatorname{Coalg}(\mathcal{T}, A b)$.

Assume $\operatorname{Tor}=\operatorname{Coalg}(\mathcal{T}, A b)$ for some Lawvere theory $\mathcal{T}$. Representing $\mathcal{T}$ as a regular quotient of some $\mathcal{T}_{\Sigma}$, we can consider $\operatorname{Coalg}(\mathcal{T}, A b)$ as an equifier in the functor category 
Coalg $Q_{\Sigma}$ for the functor $Q_{\Sigma} X=\prod_{n \in \mathbb{N}}(n \cdot X)^{\left|\Sigma_{n}\right|}=\prod_{n \in \mathbb{N}} X^{\left|n \Sigma_{n}\right|}$ according to Fact 7 Since $A b$ has biproducts the functor $A b \stackrel{Q_{\Sigma}}{\longrightarrow} A b$ preserves products, such that the forgetful functor Coalg $Q_{\Sigma} \rightarrow A b$ creates products and the equifier corresponding to $\operatorname{Coalg}(\mathcal{T}, A b)$ is closed under products. But Tor fails to be closed under products in $A b$.

\subsection{Some properties of $\operatorname{Coalg}(\mathcal{T}, \mathcal{C})$}

Proposition 16 The following hold for any cocomplete category $\mathcal{C}$ and any Lawvere theory $\mathcal{T}$.

1. $\operatorname{Coalg}(\mathcal{T}, \mathcal{C})$ has all colimits which exist in $\mathcal{C}$ and these are created by $\|-\|$.

2. Every hom-functor $\operatorname{Coalg}(\mathcal{T}, \mathcal{C}) \rightarrow$ Set has a left adjoint.

3. For every $\mathcal{S} \stackrel{\Phi}{\rightarrow} \mathcal{T}$ the functor ${ }^{\mathcal{C}} \Phi:=\operatorname{Coalg}(\mathcal{T}, \mathcal{C}) \stackrel{\mathcal{C}^{\Phi^{* o p}}}{\longrightarrow} \operatorname{Coalg}(\mathcal{S}, \mathcal{C})$ has a right adjoint ${ }_{\mathcal{C}} \Phi$.

4. The functor $\operatorname{Coalg}(\mathcal{T}, \mathcal{C}) \stackrel{{ }^{\tau} S}{\longrightarrow} \operatorname{Coalg}(\mathcal{T}, \mathcal{D})$ with respect to a finite coproduct preserving functor $\mathcal{C} \stackrel{S}{\rightarrow} \mathcal{D}$ defined as in Fact $\$ 5$, has a right adjoint $\mathcal{T} S$, provided that $S$ preserves colimits.

Proof Item 1 follows by dualization of Fact 51 and, hence Item 2 follows trivially. The right adjoint of Items 3 can be constructed by (the dual of) the familiar lifting theorem of adjunctions (see e.g [10, Chapter 4.5]). In particular, the unit of the adjunction ${ }^{\mathcal{T}} S \dashv \mathcal{T} S$ (occasionally we denote this adjunction by ${ }^{\mathcal{V}} \dashv_{\mathcal{V}} S$ if $\mathcal{T}$ is the theory of the variety $\mathcal{V}$ ) is point wise an equalizer. The same holds for the adjunction ${ }^{\mathcal{C}} \Phi \dashv \Phi_{\mathcal{C}}$.

Bergman 7] studied further categorical properties of $\operatorname{Coalg}(\mathcal{T}, \mathcal{V})$ for varieties $\mathcal{V}$ using highly technical arguments; he showed in particular that $\operatorname{Coalg}(\mathcal{T}, \mathcal{V})$ is a complete category and its underlying functor into $\mathcal{V}$ has a right adjoint. In this section we improve these results by the use of standard arguments from the theory of locally presentable categories. Hence, for the rest of this section the base category $\mathcal{C}$ in which $\mathcal{T}$-coalgebras are formed is assumed to be to be a locally presentable category.

Theorem 17 For any Lawvere theory $\mathcal{T}$ and any locally presentable category $\mathcal{C}$ the category Coalg $(\mathcal{T}, \mathcal{C})$ is locally presentable. Its forgetful functor Coalg $(\mathcal{T}, \mathcal{C}) \stackrel{\|-\|}{\longrightarrow} \mathcal{C}$ has a right adjoint $C_{\mathcal{T}, \mathcal{C}}$ and, moreover, is comonadic.

Proof The claim follows from the equivalence $\operatorname{Coalg}(\mathcal{T}, \mathcal{C}) \simeq \operatorname{Ladj}(\operatorname{Alg} \mathcal{T}, \mathcal{C})$, since for locally presentable categories $\mathcal{C}$ and $\mathcal{D}$ the category $\operatorname{Ladj}(\mathcal{C}, \mathcal{D})$ is locally presentable (see Section 1.1).

The existence of a right adjoint now follows from Item 1 of the preceding proposition by the dual of) the Special Adjoint Functor Theorem because their domains are locally presentable. Comonadicity follows by the Beck-Paré-Theorem: the category $\operatorname{Coalg}(\mathcal{T}, \mathcal{C})$, considered as a subcategory of the (comonadic) category Coalg $Q_{\Sigma}$ (see the counter example of Section 2.2 for the definition of $Q_{\Sigma}$ ), is closed under absolute equalizers.

Example 18 The cofree functor $C_{\mathcal{T}, \mathcal{C}}$ can occasionally be constructed. As an example consider the case where $\mathcal{C}={ }_{S} \operatorname{Mod}$ and $\mathcal{T}$ is the theory of ${ }_{R} \operatorname{Mod}$, hence, $\operatorname{Coalg}(\mathcal{T}, \mathcal{C})$ is the category ${ }_{S} \operatorname{Mod}_{R}$ of bimodules. Since this is a variety $\mathcal{V}$, the functor $C_{\mathcal{T}, \mathcal{C}}$ needs to be a $\mathcal{V}$-representable functor, whose representing $S$-module has, somehow, to encode the ring $R$. A natural choice, thus, is the $S$-module $F|R|$, where ${ }_{R} \operatorname{Mod} \stackrel{|-|}{\longrightarrow} A b$ denotes the forgetful functor and $A b \stackrel{F}{\rightarrow}{ }_{S} M o d$ the "free" 
functor, that is, the left adjoint of the forgetful functor $\operatorname{Mod} \rightarrow A b$, by abuse of notation also denoted by $|-|$. By the natural equivalence ${ }_{S} \operatorname{Mod}(F|R|, N) \simeq A b(|R|,|N|)$ it suffices to show that $A b(|R|,|N|)$ belongs to ${ }_{S} \operatorname{Mod}_{R}$, for every $S$-module $N$. But this obvious with $(s \cdot f)(r):=s \cdot(f(r))$ and $(f \cdot r)\left(r^{\prime}\right):=f\left(r r^{\prime}\right)$.

We note that, since ${ }_{S} \operatorname{Mod}_{R}$ is also equivalent to $\operatorname{Alg}\left(\operatorname{Mod}_{R},{ }_{S} M o d\right)$, by Fact 5 the functor $\|-\|$ also has a left adjoint. As easily seen this is given by $M \mapsto|M| \otimes_{\mathbb{Z}} R$, where the abelian group $|M| \otimes_{\mathbb{Z}} R$ inherits a left $S$-action from that of $M$, given by $s \cdot(y \otimes z):=(s \cdot y) \otimes z$, and a right $R$-action from multiplication in $R,(x \otimes y) \cdot r:=x \otimes(y r)$.

Kan already had constructed comonoids in groups on each free group [19, while Freyd has shown that every free algebra in a variety $\mathcal{V}$ carries the structure of a $\mathcal{W}$-algebra, provided there exists an algebraic functor $\mathcal{V} \rightarrow \mathcal{W}$ [16]. We now describe this construction in greater generality as follows, where $\mathcal{C} \stackrel{F}{\rightarrow} \operatorname{Alg}(\mathcal{T}, \mathcal{C})$ is the free algebra functor which exists by Fact 5 .

Proposition 19 Let $\mathcal{C}$ be a locally presentable category and $\mathcal{T}$ a Lawvere theory. Then there exists a functor $\mathcal{C} \stackrel{T}{\rightarrow} \operatorname{Coalg}(\mathcal{T}, \operatorname{Alg}(\mathcal{T}, \mathcal{C}))$ such that $\mathcal{C} \stackrel{T}{\rightarrow} \operatorname{Coalg}(\mathcal{T}, \operatorname{Alg}(\mathcal{T}, \mathcal{C})) \stackrel{\|-\|}{\longrightarrow} \operatorname{Alg}(\mathcal{T}, \mathcal{C})=$ $\mathcal{C} \stackrel{F}{\rightarrow} \operatorname{Alg}(\mathcal{T}, \mathcal{C}) . T$ has a right adjoint.

Proof By the dual of Lemma 11 the functor $\mathcal{C}(-, A)$ factors as $\mathcal{C} \rightarrow \operatorname{Alg} \mathcal{T} \stackrel{|-|}{\longrightarrow}$ Set iff $A=|\mathrm{A}|$ for a (essentially) unique $\mathcal{T}$-algebra $A$ or, in other words, for each $\mathcal{C}$-object $C$ the $\operatorname{set} \mathcal{C}(C, A)$ is the underlying set of a (essentially) unique $\mathcal{T}$-algebra $\mathrm{A}_{C}$. From the natural bijection $\operatorname{Alg}(\mathcal{T}, \mathcal{C})(F C, \mathrm{~A}) \simeq$ $\mathcal{C}(C,|\mathrm{~A}| \mathcal{C})$ one now concludes that for each $\mathcal{T}$-algebra $\mathrm{A}$ in $\mathcal{C}$ the set $\operatorname{Ag}(\mathcal{T}, \mathcal{C})(F C, \mathrm{~A})$ is the underlying set of the $\mathcal{T}$-algebra $\mathrm{A}_{C}$, such that the assignment $\mathrm{A} \mapsto \mathrm{A}_{C}$ defines a functor $\operatorname{Alg}(\mathcal{T}, C) \stackrel{R_{C}}{\longrightarrow}$ Alg $\mathcal{T}$ which is a $\mathcal{T}$-lift of $\operatorname{Alg}(\mathcal{T}, \mathcal{C})(F C,-)$. Define $T$ by $C \mapsto R_{C}$.

Since $F$ preserves and $\|-\|$ creates colimits, the functor $T$ preserves them. Thus, the final statement follows by the Special Adjoint Functor Theorem whose assumptions on the category $\mathcal{C}$ are satisfied by every locally presentable category.

\section{Coalgebras in varieties}

As from now we assume the base category $\mathcal{C}$ in which $\mathcal{T}$-coalgebras are formed to be a variety. $\mathcal{T}$-coalgebras in Alg $\mathcal{S}$ are occasionally called $\mathcal{T}$ - $\mathcal{S}$-bimodels or $\mathcal{T}$ - $\mathcal{S}$-bialgebras with the category of those denoted by $[\mathcal{T}, \mathcal{S}] .(\mathcal{T}$ - $\mathcal{T}$-bialgebras will in the sequel simply called $\mathcal{T}$-bialgebras. $)$

These categories can be used to define a bicategory having as objects all Lawvere theories, the categories $[\mathcal{T}, \mathcal{S}]$ as categories of 1-cells with horizontal composition defined by $(\mathcal{S} \stackrel{\text { B }}{\Rightarrow} \mathcal{R}) \odot(\mathcal{T} \stackrel{\text { A }}{\Rightarrow}$ $\mathcal{S}):=\mathcal{T}^{\text {op }} \stackrel{\mathrm{A}}{\rightarrow}$ Alg $\mathcal{S} \stackrel{L_{\mathrm{B}}}{\rightarrow}$ Alg $\mathcal{R}$ and the embeddings $1_{\mathcal{T}}:=Y^{\mathcal{T}}: \mathcal{T}^{\mathrm{op}} \hookrightarrow$ Alg $\mathcal{T}$ as units, and with natural transformations as 2-cells (see [4, Chapter 15]). Note that the "product" B $\odot$ A can be seen, equivalently, as the $\mathcal{T}$ - $\mathcal{R}$-bialgebra representing the composition $R_{\mathrm{A}} \circ R_{\mathrm{B}}$ of the representable functors $R_{\mathrm{A}}$ and $R_{\mathrm{B}}$, represented by $\mathrm{A}$ and $\mathrm{B}$, respectively. This construction is a straightforward generalization of the familiar bicategory $R I N G$, having as objects all unital rings (equivalently, all theories of module categories), as categories of 1-cells $[R, S]$ the categories ${ }_{R} \operatorname{Mod}_{S}$ of $R$-Sbimodules with $\otimes$ as horizontal composition, and as 2-cells the bimodule homomorphisms.

We prefer to work with the following 2-category $L A W V$, which is in view of Theorem 13 biequivalent to the bicategory just described, and where the categories of 1-cells can, alternatively, be chosen as $\operatorname{Rep}(\operatorname{Alg} \mathcal{T}, \operatorname{Alg} \mathcal{S})^{\text {op }}$. 
1. objects are all Lawvere theories,

2. the categories of 1-cells are the categories $\operatorname{Ladj}(\operatorname{Alg} \mathcal{T}, \operatorname{Alg} \mathcal{S})$ with composition of functors as horizontal composition and identities as units,

3. and 2-cells are natural transformations.

\subsection{Coalgebras in arbitrary varieties}

\subsubsection{Canonical constructions}

As follows from Proposition 19 every free algebra $F_{\mathcal{V}} X$ in a variety $\mathcal{V}$ carries the structure of a $\mathcal{V}$-coalgebra. Obviously, one then obtains a $\mathcal{W}$-coalgebra on $F_{\mathcal{V}} X$ as well, if there is an algebraic functor $\mathcal{V} \stackrel{\Phi^{*}}{\longrightarrow} \mathcal{W}$. We now will describe these structures explicitly.

Since every algebraic functor $\Phi^{*}$ induced by a morphism of Lawvere theories $\mathcal{T}_{\mathcal{W}} \stackrel{\Phi}{\rightarrow} \mathcal{T}_{\mathcal{V}}$ commutes with the underlying functors, it is $\mathcal{W}$-representable with representing object $F_{\mathcal{V}} 1$, the free $\mathcal{V}$-algebra on one generator. We denote this $\mathcal{W}$-coalgebra in $\mathcal{V}$ by $V_{\Phi}(1)$. With notations as in item 3 of Section 2.2 and item 2 of Section 2.3 one has $V_{\Phi}(1)=\mathcal{V}_{\Phi}\left(V_{\mathrm{id}}(1)\right)$. Considered as an adjunction $V_{\Phi}(1)$ is nothing but $\Phi_{*} \dashv \Phi^{*}$. These are the only $\mathcal{W}$-coalgebras on $F_{\mathcal{V}} 1$. Since Coalg $(\mathcal{W}, \mathcal{V})$ has coproducts we can form the coalgebra $V_{\Phi}(X)=X \cdot V_{\Phi}(1)$ for each set $X$. Considered as an adjunction this is $X \cdot \Phi_{*} \dashv \Phi^{* X}$ with the $\mathcal{W}$-representable functor $\Phi^{* X}$, represented by $F_{\mathcal{V}} X$. This construction is functorial since every map $X \stackrel{f}{\rightarrow} Y$ determines a natural transformation $\Phi^{*^{Y}}=\mathcal{V}\left(F_{\mathcal{V}} Y,-\right) \Rightarrow \mathcal{V}\left(F_{\mathcal{V}} X,-\right)=\Phi^{*^{X}}$, which is by the Yoneda lemma, that $F_{\mathcal{V}}(f)$ is a coalgebra morphism $V_{\Phi}(X) \rightarrow V_{\Phi}(Y)$. We so have got the following result, where the first statement is already contained in [16, and the last one follows trivially from the definition of $V_{\Phi}$ as the $X$-fold copower of $V_{\phi}(1)$ in $\operatorname{Coalg}(\mathcal{W}, \mathcal{V})$.

Theorem 20 There is an essentially one-to-one correspondence between morphism of Lawvere theories $\mathcal{T}_{\mathcal{W}} \stackrel{\Phi}{\rightarrow} \mathcal{T}_{\mathcal{V}}$ and $\mathcal{T}_{\mathcal{W}}$-coalgebra $\mathcal{V}_{\Phi}(1)$ on $F_{\mathcal{V}} 1 ;$ moreover

1. the assignment $X \mapsto X \cdot V_{\Phi}(1)$ defines a functor $V_{\Phi}: \operatorname{Set} \rightarrow \operatorname{Coalg}(\mathcal{W}, \mathcal{V})$ with $\|-\| \circ V_{\Phi}=F_{\mathcal{V}}$,

2. $V_{\Phi}$ has the functor $G_{\Phi}:=\operatorname{hom}\left(V_{\Phi}(1),-\right)$ as its right adjoint.

Remarks 21 1. With $V:=V_{\text {id }}$ the following equivalences then are obvious.

$$
\mathcal{V}_{\Phi \circ V \simeq V_{\Phi} \simeq \mathcal{W}_{\Phi_{*}} \circ W}
$$

2. The co-operations of $\mathcal{V}_{\Phi}(X)$ are the $\mathcal{V}$-homomorphisms $F_{\mathcal{V}} X \stackrel{X \cdot \Phi(\sigma)}{\longrightarrow} X \cdot F_{\mathcal{V}} n \simeq n \cdot F_{\mathcal{V}} X$ for all $\sigma \in \mathcal{T}(n, 1)=\mathcal{W}\left(F_{\mathcal{W}} 1, F_{\mathcal{W}} n\right)$ and $n \in \mathbb{N}$. These co-operations act on the free generators $x \in X$ of $F_{\mathcal{V}} X$ as $X \stackrel{\eta_{X}}{\longrightarrow} F_{\mathcal{V}} X \stackrel{\left\langle\nu_{k}\right\rangle}{\longrightarrow}\left(F_{\mathcal{V}}(n \cdot X)\right)^{n} \stackrel{\Phi(\sigma)^{F_{\mathcal{V}}(n \cdot X)}}{\longrightarrow} F_{\mathcal{V}}(n \cdot X) \simeq n \cdot F_{\mathcal{V}} X$, where $\eta_{X}$ is the insertion of generators and $\left\langle\nu_{k}\right\rangle$ is the homomorphism whose coordinates are the coproduct injections $F_{\mathcal{V}} X \stackrel{\nu_{k}}{\longrightarrow} n \cdot F_{\mathcal{V}} X$. In other words,

$$
\sigma_{V_{\Phi}(X)} \circ \eta_{X}=\Phi(\sigma)^{F_{\mathcal{V}}(n \cdot X)} \circ\left\langle\nu_{k}\right\rangle \circ \eta_{X}
$$


This situation is illustrated by the following diagram, where the unlabelled arrows are the forgetful functors. Here all cells obviously commute, while commutativity of the outer frame represents Equation 10 .

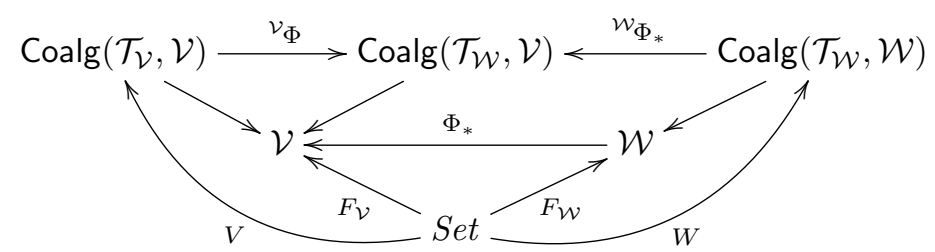

A concrete description of the right adjoint of $V_{\Phi}$ is obtained as follows where, for $\tau \in \mathcal{T}_{\mathcal{W}}(n, 1)$, we denote by $\tau_{\mathrm{A}}$ the underlying map of the $\mathcal{V}$-homomorphism $\mathrm{A} \tau$, by $\Phi(\tau)^{n \cdot A}$ the interpretation of $\Phi \tau$ in the $\mathcal{V}$-algebra $n \cdot A$, and by $\nu_{1}, \ldots, \nu_{n}: A \rightarrow n \cdot A$ in $\mathcal{V}$ the coproduct injections.

Proposition 22 For every $\mathcal{T}_{\mathcal{W}}$-coalgebra $\mathrm{A}$ in $\mathcal{V}$ the set $G_{\Phi}(\mathrm{A})$ is the subset

$$
G_{\Phi} \mathrm{A}=\bigcap_{t \in \mathcal{T}_{\mathcal{W}}(n, 1), n \in \mathbb{N}} \operatorname{Eq}_{S e t}\left(t_{\mathrm{A}},(\Phi(t))^{n \cdot A} \circ\left\langle\nu_{k}\right\rangle\right)
$$

of $|\|\mathrm{A}\||$ and the embeddings $e_{\mathrm{A}}$ form are a natural transformation $e: G_{\Phi} \Rightarrow|-| \circ\|-\|$.

Proof Identifying $\operatorname{Coalg}\left(\mathcal{T}_{\mathcal{W}}, \mathcal{V}\right)$ and $\operatorname{Adj}\left(\operatorname{Alg} \mathcal{T}_{\mathcal{W}}, \mathcal{V}\right)$, the hom-set $\operatorname{hom}\left(V_{\Phi}(1), \mathrm{A}\right)$ is $\operatorname{nat}\left(\Phi_{*}, L_{\mathrm{A}}\right)$, the set of natural transformations from $\Phi_{*}$ into the left adjoint $L_{\mathrm{A}}$ of $\mathrm{A}$ corresponding to $\mathrm{A}$, for each $\mathcal{T}_{\mathcal{W}}$-coalgebra A. Given a natural transformation $\mu: \Phi_{*} \Rightarrow L_{\mathrm{A}}$, one has $\|\mu\|=\mu_{1}: \Phi_{*}\left(F_{\mathcal{W}} 1\right)=$ $F_{\mathcal{V}} 1 \rightarrow L_{\mathrm{A}} F_{\mathcal{W}} 1=\mathrm{A}(1)=|\mathrm{A}|$; hence, the assignment $\mu \mapsto \mu_{1}(1)$ defines an injective map $G_{\Phi}(\mathrm{A}) \stackrel{e_{\mathrm{A}}}{\longrightarrow}|\|\mathrm{A}\||$ and these maps form a natural transformation, trivially.

Equation 12 is equivalent to the following statement: For every $\mathcal{V}$-homomorphisms $F_{\mathcal{V}} 1 \stackrel{f}{\rightarrow}$ $A=\mathrm{A}(1)$ the family $(n \cdot f)_{n}$ is a natural transformation $\Phi_{*} \Rightarrow L_{\mathrm{A}}$ if and only if $f(1) \in G_{\Phi} \mathrm{A}$. But this is evident by the following diagram, using Equation (11).

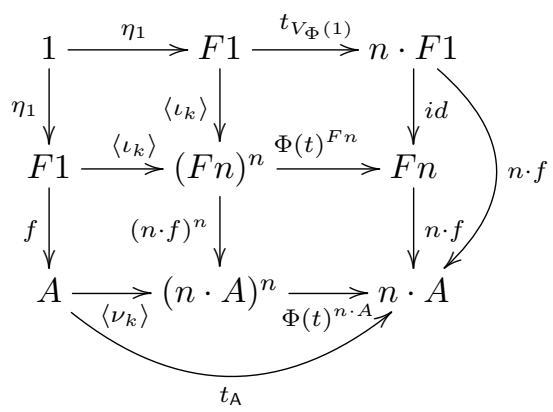

Remarks 23 The following facts are easy to verify.

1. $G_{\Phi} \mathrm{A}$ contains all $\mathcal{V}$-constants in $A$.

2. By Equation (11) for every set $X$ the following holds: $X \subset G_{\Phi} V_{\Phi}(X) \subset\left|F_{\mathcal{V}} X\right|$. In other words the set $G_{\Phi} V_{\Phi}(X)$ generates the $\mathcal{V}$-algebra $\left\|V_{\Phi}(X)\right\|$. In general this is not the case for arbitrary coalgebras and this implies that $G_{\Phi}$ in general fails to be faithful. 
3. If $\mathcal{T}_{\Sigma} \stackrel{[-]}{\longrightarrow} \mathcal{T}_{\mathcal{W}}$ is a regular quotient, then

$$
G_{\Phi} \mathrm{A}=\bigcap_{\sigma \in \Sigma_{n}, n \in \mathbb{N}} \operatorname{Eq}_{S e t}\left(\sigma_{\mathrm{A}},(\Phi \sigma)^{n \cdot A} \circ\left\langle\nu_{k}\right\rangle\right) .
$$

4. Writing $G_{\mathcal{V}}$ instead of $G_{\mathrm{id} \mathcal{V}}$ commutativity of Diagram 3.1.1) implies by composition of adjunctions

(a) The forgetful functor $\mathcal{W} \stackrel{|-|}{\longrightarrow}$ Set factors as $\mathcal{W} \stackrel{C_{\mathcal{T}_{\mathcal{W}}, \mathcal{W}}}{\longrightarrow} \operatorname{Coalg}\left(\mathcal{T}_{\mathcal{W}}, \mathcal{W}\right) \stackrel{G_{\mathcal{W}}}{\longrightarrow}$ Set and

(b) $\operatorname{Coalg}\left(\mathcal{T}_{\mathcal{W}}, \mathcal{V}\right) \stackrel{G_{\Phi}}{\longrightarrow}$ Set factors as $\operatorname{Coalg}\left(\mathcal{T}_{\mathcal{W}}, \mathcal{V}\right) \stackrel{\mathcal{T}_{\mathcal{W}} \Phi_{*}}{\longrightarrow} \operatorname{Coalg}\left(\mathcal{T}_{\mathcal{W}}, \mathcal{W}\right) \stackrel{G_{\mathcal{W}}}{\longrightarrow}$ Set and as $\operatorname{Coalg}\left(\mathcal{T}_{\mathcal{W}}, \mathcal{V}\right) \stackrel{\mathcal{V}_{\Phi}}{\longrightarrow} \operatorname{Coalg}\left(\mathcal{T}_{\mathcal{V}}, \mathcal{V}\right) \stackrel{G_{\mathcal{V}}}{\longrightarrow} \operatorname{Set}$

Lemma 24 Given a factorization $\Phi=\mathcal{T}_{\mathcal{W}} \stackrel{\Xi}{\rightarrow} \mathcal{T}_{\mathcal{U}} \stackrel{\Sigma}{\rightarrow} \mathcal{T}_{\mathcal{V}}$ in Lawv, the following diagrams commute.
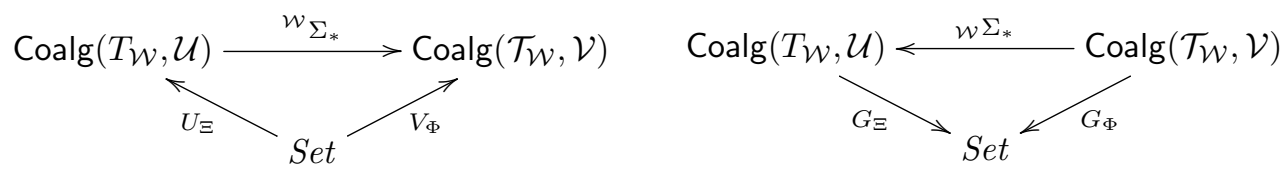

Example 25 With notations as in Diagram (9) Coalg $\left(M o n,{ }_{c} A l g_{R}\right)$ is equivalent to the category ${ }_{c}$ Bialg $_{R}$ of all commutative unital and counital $R$-bialgebras, while Coalg $\left(A b,{ }_{c} A l g{ }_{R}\right)$ is equivalent to the category ${ }_{b i} \operatorname{Hopf}_{R}$ of all bicommutative $R$-Hopf algebras.

By Equation 12. one gets, for any commutative bialgebra $\mathrm{A}=(A, m, e, \Delta, \epsilon)$,

$$
G_{\Phi}(\mathrm{A})=\{a \in A \mid \Delta(a)=a \otimes 1+1 \otimes a\}
$$

that is, $G_{\Phi}(\mathrm{A})$ is the usual set of primitive elements of $\mathrm{A}$, while for any bicommutative bialgebra

$$
G_{\Psi}(\mathrm{A})=\{a \in A \mid \Delta(a)=a \otimes a, \epsilon(a)=e(1)\}
$$

is the usual set of group-like elements of $A$.

For ${ }_{R} \operatorname{Mod}_{R} \simeq$ Coalg $\left({ }_{R} \operatorname{Mod},{ }_{R} M o d\right)$, the category of $R$-bimodules, and any bimodule $M$

$$
G(M)=\{m \in M \mid r m=m r \quad \forall r \in R\}
$$

is the space of $R$-invariants of $M$ in the sense of [5].

Remark 26 (Comonoids in the category of groups) With $\mathcal{V}=G r p, \mathcal{W}=M o n$ and the forgetful functor $\Phi^{*}: \mathcal{V} \rightarrow \mathcal{W}$ the following has been shown by Kan (see [19]): Every comonoid in Grp has as its underlying group a free group $F X$ and every free group carries precisely one comonoid structure ([19, Thm. 3.10]). This theorem states in our terminology (with $X_{\mathrm{A}}:=$ $\left.G_{\Phi} \mathrm{A} \backslash\{1\}\right)$ : For every comonoid $\mathrm{A}$ in Grp there is an isomorphism $\mathrm{A} \simeq V_{\Phi}\left(X_{\mathrm{A}}\right)$.

A then is an internal cogroup in Grp ([19, Ex. 6.5]), which is in our terminology $V\left(X_{\mathrm{A}}\right)$; this is clear by Remarks 21, the co-inversion of any cogroup $V(X)$ is the homomorphic extension $F X \stackrel{\iota}{\rightarrow} F X$ of the map $X \rightarrow F X$ with $x \mapsto x^{-1}$, where $x^{-1}$ denotes the inverse of $x$ in the free group $F X$.

This result implies that the embedding $\Phi^{*}$ is the only algebraic functor from Grp to Mon and the identity is the only algebraic functor on Grp. Combining Kan's result with the methods developed above we can give concrete descriptions of the following constructions of which we so far only know (see Theorem 17) that they exist (cp. [7, pp 399-400]): 
1. A right adjoint of the forgetful functor $\operatorname{Coalg}(\operatorname{Grp}, \operatorname{Grp}) \stackrel{\|-\|}{\longrightarrow} \operatorname{Grp}$ is given by the functor $\operatorname{Grp} \stackrel{|-|}{\longrightarrow}$ Set $\stackrel{V}{\longrightarrow}$ Coalg $(G r p, G r p)$. In fact, the counit of the free group adjunction $\|V(|A|)\|=F|A| \stackrel{\epsilon_{A}}{\longrightarrow} A$ is the required counit: For any group homomorphism $\|\mathrm{B}\|=$ $\left\|V\left(X_{\mathrm{B}}\right)\right\|=F\left(X_{\mathrm{B}}\right) \stackrel{f}{\rightarrow} A$ the homomorphism $F X_{\mathrm{B}} \stackrel{F(f)}{\longrightarrow} F|A|$ is the only homomorphism $f^{*}$ with $\epsilon_{|A|} \circ f^{*}=f$ and coincides with $\|V(|f|)\|$.

2. Products in Coalg $(G r p, G r p)$ can be constructed as follows, since Coalg $(\operatorname{Grp}, \operatorname{Grp}) \stackrel{\text { G }}{\rightarrow}$ Set is a right adjoint of $V$ and, hence, preserves products (while $V$ preserves coproducts):

Let $\mathrm{A}$ and $\mathrm{B}$ be cogroups in Grp. Writing $\mathrm{A}=V\left(X_{\mathrm{A}}\right)$ and $\mathrm{B}=V\left(X_{\mathrm{B}}\right)$ we have $X_{\mathrm{A} \times \mathrm{B}}=$ $G_{\mathcal{V}}(\mathrm{A} \times \mathrm{B})-1=G_{\mathcal{V}} \mathrm{A} \times G_{\mathcal{V}} \mathrm{B}-1=\left(X_{\mathrm{A}}+1\right) \times\left(X_{\mathrm{B}}+1\right)-1$ and, thus,

$$
\mathrm{A} \times \mathrm{B}=V\left(X_{\mathrm{A}} \times X_{\mathrm{B}}+X_{\mathrm{A}}+X_{\mathrm{B}}\right)=V\left(X_{\mathrm{A}} \times X_{\mathrm{B}}\right)+\mathrm{A}+\mathrm{B} .
$$

Kan's result is a rather special property of the variety Grp and its embedding into Mon. As follows from Remark 12 and the discussion of linear groups in Section 3.1 .2 below, in general there exist coalgebras on non-free algebras as well, and free algebras may allow for more than one coalgebra structure. The examples mentioned in Example 31 below share with Grp the property that the coalgebras $V(X)$ in $\mathcal{V}$ are the only $\mathcal{V}$-coalgebras in $\mathcal{V}$; as the argument above shows also in these cases $\|-\|$ is left adjoint to $V \circ|-|$. In view of Item 2 of Remarks 23 they provide however the other extreme case of the possible sizes of the sets $G_{\mathcal{V}} V(X)$ : while for $\mathcal{V}=G r p$ the set $G_{\mathcal{V}} V(X)$ is the smallest possible one, in those cases it is the largest.

\subsubsection{Some applications}

Change of rings. Consider a ring homomorphism $R \stackrel{\phi}{\rightarrow} S$ (equivalently, a morphism of Lawvere theories $\Phi: \mathcal{T}_{R \text { Mod }} \rightarrow \mathcal{T}_{S \text { Mod }}$.

The algebraic functor $\Phi^{*}: \mathcal{V}={ }_{S} \operatorname{Mod} \rightarrow{ }_{R} \operatorname{Mod}$ is the ${ }_{R} \operatorname{Mod}$-representable functor ${ }_{S} \operatorname{hom}(S,-)$, known as the restriction of scalars-functor.

The corresponding $S$-left, $R$-right bimodule structure on $S$ is $V_{\Phi}(1)$. By the above its left adjoint $\Phi_{*}$ is the functor $V_{\Phi}(1) \otimes_{R}-$, known as the extension of scalars-functor.

Since the forgetful functors from module categories into $A b$ create colimits, the functor $\Phi^{*}$, being a concrete functor over $A b$, preserves them. Consequently, the restriction of $\Phi^{*}$ to the dual of the theory of ${ }_{S} M o d$ is an ${ }_{S}$ Mod-coalgebra in ${ }_{R} M o d$ and $\Phi^{*}$ is its left Kan

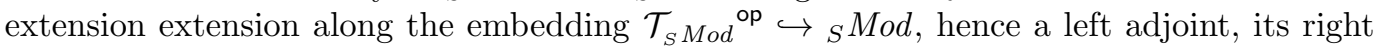
adjoint $G$ being the ${ }_{S} M o d$-lift of ${ }_{R} \operatorname{hom}\left(\Phi^{*} S,-\right)$. In other words, $G$ is the coextension of scalars-functor.

We so have obtained, without any calculations, the familiar adjunctions extension of scalars $\dashv$ restriction of scalars $\dashv$ coextension of scalars.

Morita theory. Lawvere theories $\mathcal{T}$ and $\mathcal{S}$ are called Morita equivalent if the categories $\mathrm{Alg} \mathcal{T}$ and Alg $\mathcal{S}$ are equivalent (not necessarily concretely so). Since an equivalence $L$ is a left and a right adjoint of its equivalence inverse $R$ one concludes from Theorem 13 that the following are equivalent, where the respective coalgebras $\mathrm{A}$ and $\mathrm{B}$ are related by the property $L_{\mathrm{A}} \simeq R_{\mathrm{B}}$ and $L_{\mathrm{B}} \simeq R_{\mathrm{A}}$.

i. $\operatorname{Alg} \mathcal{T} \stackrel{L}{\rightarrow} \operatorname{Alg} \mathcal{S}$ is an equivalence with equivalence inverse $\operatorname{Alg} \mathcal{S} \stackrel{R}{\rightarrow} \operatorname{Alg} \mathcal{T}$. 
ii. There exists a $\mathcal{T}$-coalgebra $\mathrm{A}$ in $\operatorname{Alg} \mathcal{S}$ with $L \simeq L_{\mathrm{A}}$ and $R \simeq R_{\mathrm{A}}$.

iii. There exists a $\mathcal{S}$-coalgebra $\mathrm{B}$ in $\operatorname{Alg} \mathcal{T}$ with $R \simeq L_{\mathrm{B}}$ and $L \simeq R_{\mathrm{B}}$.

Thus, Lawvere theories $\mathcal{T}$ and $\mathcal{S}$ are Morita equivalent, if they are equivalent in the 2category $L A W V$.

The coalgebras $A$ and $B$ above can in view of Section 1.2 .3 equivalently be described as follows: $\mathrm{A}(1)$ is a varietal generator in $\mathrm{Alg} \mathcal{S}$ such that $\mathcal{T} \simeq\left(\mathcal{T}_{(\mathrm{Alg} \mathcal{S})^{\text {op }}}[\mathrm{A}(1)]\right)$ and $\mathrm{B}(1)$ is a varietal generator in $\operatorname{Alg} \mathcal{T}$ such that $\mathcal{S} \simeq\left(\mathcal{T}_{(\operatorname{Alg} \mathcal{T})^{\circ p}}[\mathrm{~B}(1)]\right)$. This follows from the facts that $\mathrm{A}(1)=L\left(F_{\mathrm{Alg} \mathcal{T} 1}\right)$ is a varietal generator in $\operatorname{Alg} \mathcal{S}$ since $F_{\mathrm{Alg} \mathcal{T} 1}$ is a varietal generator in $\operatorname{Alg} \mathcal{T}$ and equivalences clearly preserve varietal generators, and $A$, being a restriction of the equivalence $L_{\mathrm{A}}$, is full and faithful (analogously for B).

As is well known every variety equivalent to a variety ${ }_{R} \operatorname{Mod}$ is necessarily of the form ${ }_{S}$ Mod since module categories can be characterized as those varieties which are Abelian categories and this property is preserved by equivalences. (Algebraically one may argue as follows: since every (varietal) generator $G$ in ${ }_{R} M o d$ is a $R$-S-bimodule for $S=\operatorname{End}(G)$, the endomorphism ring of $G$ (see e.g. [6, Theorem 17.8]), every variety $\mathcal{V}$, which is equivalent to ${ }_{R} M o d$ is concretely equivalent to ${ }_{S} M o d$.) Hence, one can restrict the above to the bicategory $R I N G$ of rings with bimodules as 1-cells and so obtains the classical Morita theory as an immediate consequence. The varietal generators in ${ }_{R} M o d$ are the so-called progenerators.

General and special linear groups. Assigning to a commutative unital ring $R$ the monoid $M(n, R)$ of $n \times n$-matrices over $R$, the general linear group $G L(n, R)$ or the special linear group $S L(n, R)$ defines functors $M_{n}:$ Ring $\rightarrow$ Mon, GL $L_{n}:$ Ring $\rightarrow$ Grp, and $S L_{n}:$ Ring $\rightarrow$ Grp , respectively. These functors are Mon- and Grp-representable, respectively and, hence, all have a left adjoint. They do not arise from the canonical constructions.

Since $\mathbb{N}$ is the free monoid over a singleton $\{\star\}$ it suffices to find a $M_{n}$-universal monoid homomorphism $\mathbb{N} \stackrel{u}{\rightarrow} M\left(n, A_{n}\right)$. Choose $A_{n}$ to be $\mathbb{Z}\left[X_{i, j} ; 1 \leq i, j \leq n\right]$, the free commutative ring $F n^{2}$ over $n^{2}$ and $u$ the monoid morphism mapping 1 to the $n \times n$ identity matrix $E_{n}=\left(\delta_{i j}\right)$ over $A_{n}$. For every $M \in M(n, R)$ we have the unique ring homomorphism $\psi: \mathbb{Z}\left[X_{i, j}\right] \rightarrow R$ with $\delta_{i j} \mapsto m_{i j}$. Then $M_{n}(\psi)=M$ and $\psi$ is unique with this property. Thus, $M_{n}$ is Mon-representable with representing object $F n^{2}$.

Since $G L_{n}=\operatorname{Ring} \stackrel{M_{n}}{\longrightarrow}$ Mon $\stackrel{(-)^{\times}}{\longrightarrow} \operatorname{Grp}$ and $(-)^{\times}$is right adjoint to the forgetful functor $G r p \rightarrow$ Mon the functor $G L_{n}$ is $G r p$-representable with representing object $F n^{2}$.

Concerning $S L_{n}$ one proceeds analogously. One needs to find a $S L_{n}$-universal group homomorphism $\mathbb{Z} \stackrel{u}{\rightarrow} S L\left(n, A_{n}\right)$. Consider the coequalizer $F\left(n^{2}\right) \stackrel{q}{\rightarrow} A_{n}$ of the ring homomorphisms $\operatorname{det}^{\sharp}, 1^{\sharp}: F 1 \rightarrow F n^{2}$ given by det and the constant 1 , considered as $n^{2}$-ary derived operations (hence elements of $F n^{2}$ ) in the theory of commutative rings. It then is easy to see that the group homomorphism $u$ mapping $1 \in \mathbb{Z}$ to the $n \times n$ identity matrix $\left(\delta_{i j}\right)$ over $A_{n}$ does the job.

In fact Theorem 13 more generally implies that every affine group scheme, that is, every Grp-representable functor on ${ }_{c} \mathrm{Alg}_{R}$, for a commutative ring R, has a left adjoint.

The primitive Hopf structure on polynomial algebras. It is well known that, for every commutative ring $R$, the polynomial algebras in ${ }_{c} A l g_{R}$, that is, the free algebras in ${ }_{c} A l g_{R}$, carry the structure of an $R$-Hopf algebra in which the variables, that is, the free generators, are primitive elements (see e.g. [1, p. 92]). Recalling that the category ${ }_{b i} H_{o p f_{R}}$ of bicommutative 
Hopf algebras over $R$ is essentially nothing but $\operatorname{Coalg}\left(A b,{ }_{c} A l g_{R}\right)$ (see Section 2.2), one gets the functor $S e t \stackrel{V_{\Phi}}{\longrightarrow}{ }_{b i} H_{o p f}$ determined by the algebraic functor ${ }_{c} A l g_{R} \stackrel{\Phi^{*}}{\longrightarrow} A b$, assigning to a set the respective polynomial algebra.

Thus, item 2 of Remarks 23 explains the result mentioned at the beginning of this example, since $G_{\Phi} H$ is the set of primitive elements of a bicommutative Hopf algebra $H$ (see Example 25).

Adjoint monads induced by bialgebras. Any $\mathcal{T}$-bialgebra $\mathrm{A}$, that is, any 1-cell in $L A W V$ may, as in any bicategory, allow for the structure of a monad $\mathbb{A}=\left(\mathrm{A}, Y^{\mathcal{T}} \stackrel{\eta}{\rightarrow} \mathrm{A}, \mathrm{A} \odot \mathrm{A} \stackrel{\mu}{\rightarrow} \mathrm{A}\right)$. Such monad is the same as a monoid in the monoidal category $\left([\mathcal{T}, \mathcal{T}], \odot, Y^{\mathcal{T}}\right)$ obtaining its monoidal structure from the monoidal structure of $(\operatorname{Ladj}(\operatorname{Alg} \mathcal{T}, \operatorname{Alg} \mathcal{T}), \circ, i d)$ by the equivalence of Theorem 13 . The monad $\mathbb{A}$, considered as a monad in $(\operatorname{Ladj}(\operatorname{Alg} \mathcal{T}, \operatorname{Alg} \mathcal{T}), \circ, i d)$, is a usual monad $\left(L_{\mathrm{A}}, L_{\mathrm{A}} \circ L_{\mathrm{A}} \stackrel{\mu}{\rightarrow} L_{\mathrm{A}}, i d_{\mathrm{Alg} \mathcal{T}} \stackrel{\eta}{\rightarrow} L_{\mathrm{A}}\right)$ on $\operatorname{Alg} \mathcal{T}$ (not to be confused with the monad $\mathbb{A}$ on $S e t$ of Section 2.1.4 and so determines its Eilenberg-Moore category $(\operatorname{Alg} \mathcal{T})^{\mathbb{A}}$. It is not difficult to see that the composition of forgetful functors $(\operatorname{Alg} \mathcal{T})^{\mathbb{A}} \rightarrow \operatorname{Alg} \mathcal{T} \rightarrow$ Set is a finitary monadic functor such that one can identify the category $(\operatorname{Alg} \mathcal{T})^{\mathbb{A}}$ with $\operatorname{Alg} \mathcal{T}_{\mathbb{A}}$ for some Lawvere theory $\mathcal{T}_{\mathbb{A}}$ (see e.g. [4, A.21]). The forgetful functor of Alg $\mathcal{T}_{\mathbb{A}} \rightarrow$ Set factors as $\operatorname{Alg} \mathcal{T}_{\mathbb{A}} \simeq(\operatorname{Alg} \mathcal{T})^{\mathbb{A}} \rightarrow \operatorname{Alg} \mathcal{T} \stackrel{|-|}{\longrightarrow}$ Set, where the functor $\operatorname{Alg} \mathcal{T}_{\mathbb{A}} \stackrel{U_{\mathbb{A}}}{\longrightarrow}$ Alg $\mathcal{T}$ is finitary monadic; since $U_{\mathbb{A}}$ commutes with the forgetful functors it is an algebraic functor and so determines a theory morphism $\mathcal{T} \stackrel{\Phi}{\rightarrow} \mathcal{T}_{\mathbb{A}}$. Categories of the form $\operatorname{Alg} \mathcal{T}_{\mathbb{A}}$ first appeared in 28 with $\mathcal{T}$ the category of commutative rings; here also applications of this construction are given.

Recall from [15]: If $F \dashv U$ be an adjunction from $\mathcal{C}$ to $\mathcal{D}$, whose induced monad on $\mathcal{C}$ is $\mathbb{A}$, then the following conditions are equivalent,

1. There exists an adjunction $U \dashv G$, inducing a comonad $\mathbb{C}$ on $\mathcal{C}$.

2. There exists an adjunction $T:=U F \dashv C:=U G$.

and imply that the (co)Eilenberg-Moore categories $\mathcal{C}^{\mathbb{A}}$ and $\mathcal{C}_{\mathbb{C}}$ coincide up to a concrete isomorphism over $\mathcal{C}$ and, hence, $U$ is monadic and comonadic.

Applying this to the above, with $\mathcal{C}=\operatorname{Alg} \mathcal{T}, U_{\mathbb{A}}=\Phi^{*}$, and $F=\Phi_{*}$ we see, since $L_{\mathrm{A}}=U_{\mathbb{A}} F=$ $\Phi^{*} \circ \Phi_{*}$ has the right adjoint $R_{\mathrm{A}}$, that the functor $U_{\mathbb{A}}=\Phi^{*}$ is monadic and comonadic. We so obtain the following characterization of theory morphisms, whose induced algebraic functor has a right adjoint where the equivalence of 1 . and 2 . is well known.

Equivalent are, for any theory morphisms $\mathcal{T} \stackrel{\Phi}{\rightarrow} \mathcal{S}$,

1. $\Phi^{*}$ has a right adjoint $R$.

2. $\Phi^{*}$ is monadic and comonadic.

3. The $\mathcal{T}$-bialgebra A corresponding to the left adjoint functor $L:=\Phi^{*} \circ \Phi_{*}$ (equivalently, representing the right adjoint $\left.R \circ \Phi^{*}\right)$ carries a monad $\mathbb{A}$ such that $\mathcal{S} \simeq \mathcal{T}_{\mathbb{A}}$ and $\Phi^{*}=U_{\mathbb{A}}$.

The following equivalence, for any $\mathcal{T}$-bialgebra $A$, characterizes the monad structures on left adjoint endofunctors of varieties in terms of their right adjoints.

$L_{\mathrm{A}}$ carries the structure of a monad $\mathbb{A} \Longleftrightarrow$ The functor $R_{\mathrm{A}}$ has a right adjoint 
We illustrate the above by the following simple example: Consider the functors Mon $\stackrel{(-)^{\times}}{\longrightarrow}$ $\operatorname{Grp} \stackrel{\Lambda^{*}}{\longrightarrow}$ Mon of Diagram (9). Here $\Lambda^{*}$ has $(-)^{\times}$as its right adjoint. $(-)^{\times}$is the Grp-lift of the functor $\operatorname{Mon}((\mathbb{Z},+, 0),-)$ and $\Lambda^{*} \circ(-)^{\times}$is the Mon-lift of $\operatorname{Mon}((\mathbb{Z},+, 0),-)$.

Grp not only is concretely equivalent to the category $M_{0} n^{\mathbb{T}}$ for the monad $\mathbb{T}$ given by the adjunction $\Lambda_{*} \dashv \Lambda^{*}$, but also to Mon $_{\mathbb{A}}$ for the comonad $\mathbb{A}$ given by the adjunction $(-)^{\times} \vdash \Lambda^{*}$ : a monoid $M$ carries a (unique) group structure iff $M \simeq \Lambda^{*}\left(M^{\times}\right)$that is, in other words, groups are the coalgebras of the comonad $\mathbb{A}$, since the counit of the adjunction $(-)^{\times} \vdash \Lambda^{*}$ is an isomorphism.

\subsection{Coalgebras in commutative varieties}

A Lawvere theory $\mathcal{T}$ is called commutative, if for all $\sigma \in \mathcal{T}(n, 1), \tau \in \mathcal{T}(m, 1)$ the following diagram commutes.

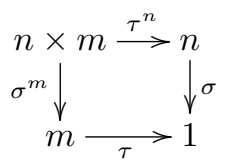

Varieties $\mathcal{V}$ whose Lawvere theory $\mathcal{T}_{\mathcal{V}}$ is commutative are known under various names as, e.g., entropic varieties, distribute varieties or commutative varieties (see 12 for the respective references). Though the first option seems to be the more popular choice amongst universal algebraists, we will use the second one because of its match with the notion of a commutative theory. We notice that for a commutative theory $\mathcal{T}$ the categories $\operatorname{Alg}(\mathcal{T}, \operatorname{Alg} \mathcal{T})$ and $\operatorname{Alg} \mathcal{T}$ are equivalent.

Since limits in functor categories are computed point wise one immediately gets from this definition, that for any commutative Lawvere theory $\mathcal{T}$ and for every internal $\mathcal{T}$-algebra $\mathrm{A}$ in a category $\mathcal{C}$ with finite products the $\mathrm{A}$ interpretation $\mathrm{A} \tau$ of any $m$-ary operation $\tau \in \mathcal{T}(m, 1)$ is a morphism $\mathrm{A}^{m} \rightarrow \mathrm{A}$ in $\operatorname{Alg}(\mathcal{T}, \mathcal{C})$, that is, for every $\sigma \in \mathcal{T}(n, 1)$ and every $n \in \mathbb{N}$ the following diagram commutes in $\mathcal{V}$, where $A:=\mathrm{A} 1$.

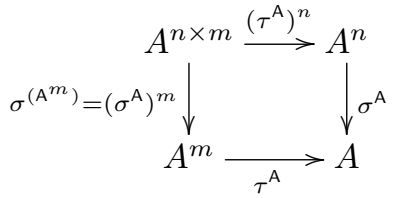

By dualization we obtain

Proposition 27 Let $\mathcal{T}$ be a commutative Lawvere theory. Then for every variety $\mathcal{V}$ the category $\operatorname{Coalg}(\mathcal{T}, \mathcal{V})$ is cocommutative, that is, for every $\mathcal{T}$-algebra $\mathcal{T} \stackrel{\mathrm{A}}{\rightarrow} \mathcal{V}^{\mathrm{op}}$ and every $\tau \in \mathcal{T}(m, 1)$ the $\mathcal{V}$-homomorphism $\mathrm{A} \tau=A \stackrel{\tau_{\mathrm{A}}}{\longrightarrow} m \cdot A$ is a morphism in $\operatorname{Coalg}(\mathcal{T}, \mathcal{V})$.

\subsubsection{Canonical coalgebras}

Since for a commutative variety $\mathcal{V}$ we have the equivalence $\operatorname{Alg}\left(\mathcal{T}_{\mathcal{V}}, \mathcal{V}\right) \simeq \mathcal{V}$, the functor $T$ of Proposition 19 is an equivalence and, hence the functor $T$ is a coreflective embedding. Again, in this situation a simpler description of this fact can be given as follows.

In a commutative variety $\mathcal{V}$ the hom-sets $\mathcal{V}(A, B)$ form subalgebras $[A, B]$ of the products $B^{A}$. $\mathcal{V}$ then is a monoidal closed category with internal hom-functor $[-,-]$ so defined (see [12, 10]). In particular, for each $\mathcal{V}$-algebra $A$ one has an adjunction $A \otimes-\dashv[A,-]$ on $\mathcal{V}$ and, thus, a canonical 
$\mathcal{T}_{\mathcal{V}}$-coalgebra $N A$ with underlying $\mathcal{V}$-algebra $A$. Equivalently, $N A$ is the $\mathcal{T}_{\mathcal{V}}$-coalgebra in $\mathcal{V}$ with corresponding $\mathcal{T}_{\mathcal{V}}$-representable functor $R_{N A}=\mathcal{V} \stackrel{[A,-]}{\longrightarrow} \mathcal{V}$. The co-operation $A \stackrel{\sigma_{N A}}{\longrightarrow} n \cdot A$ of $N A$ corresponding to $\sigma \in \mathcal{T}_{\mathcal{V}}(n, 1)$ is (use Equation (11) and the fact that operations in $\mathcal{V}$ are homomorphisms and Theorem 13 respectively)

$$
A \stackrel{\sigma_{N A}}{\longrightarrow} n \cdot A=\left(A \simeq A \otimes F_{\mathcal{V}} 1 \stackrel{A \otimes \sigma}{\longrightarrow} A \otimes F_{\mathcal{V}} n \simeq n \cdot A\right)=A \stackrel{\left\langle\nu_{1}, \ldots, \nu_{n}\right\rangle}{\longrightarrow}(n \cdot A)^{n} \stackrel{\sigma^{n \cdot A}}{\longrightarrow} n \cdot A
$$

This defines a full embedding $N: \mathcal{V} \rightarrow \operatorname{Coalg}\left(\mathcal{T}_{\mathcal{V}}, \mathcal{V}\right)$ with $\|-\| \circ N=i d_{\mathcal{V}}$. Though $N$ occasionally is an equivalence, that is, up to isomorphism $\mathcal{V}$ only admits canonical $\mathcal{T}_{\mathcal{V}}$-coalgebras, this is not the case general (see Examples 31 below). Slightly more general we have the following results.

Theorem 28 Let $\Phi: \mathcal{T} \rightarrow \mathcal{T}_{\mathcal{V}}$ be a theory morphism into a commutative theory $\mathcal{T}_{\mathcal{V}}$. Then the following hold, with notation as above:

1. The functor $N_{\Phi}=\mathcal{V} \stackrel{N}{\longrightarrow} \operatorname{Coalg}\left(\mathcal{T}_{\mathcal{V}}, \mathcal{V}\right) \stackrel{\mathcal{V}_{\Phi}}{\longrightarrow} \operatorname{Coalg}(\mathcal{T}, \mathcal{V})$ is full and faithful.

2. $N_{\Phi}\left(F_{\mathcal{V}} X\right)=V_{\Phi}(X)$ and $\|-\| \circ N_{\Phi}=\mathrm{id}_{\mathcal{V}}$.

3. The assignment $\Phi \mapsto N_{\Phi}$ defines an essentially bijective correspondence between morphisms of Lawvere theories $\Phi: \mathcal{T} \rightarrow \mathcal{T}_{\mathcal{V}}$ and functors $S: \mathcal{V} \rightarrow \operatorname{Coalg}(\mathcal{T}, \mathcal{V})$ with $\|-\| \circ S=\mathrm{id}_{\mathcal{V}}$.

4. The $\mathcal{T}$-representable functor corresponding to $N_{\Phi} A$ is $R_{N_{\Phi} A}=\mathcal{V} \stackrel{[A,-]}{\longrightarrow} \mathcal{V} \stackrel{\Phi^{*}}{\longrightarrow} A \lg \mathcal{T}$, for any $\mathcal{V}$-algebra $A$. The co-operation $\sigma_{N_{\Phi} A}$ corresponding to $\sigma \in \mathcal{T}(n, 1)$ is explicitly given by $\sigma_{N_{\Phi} A}=\Phi(\sigma)^{n \cdot A} \circ\left\langle\nu_{1}, \cdots, \nu_{n}\right\rangle$.

Proof Considering coalgebras as representable functors $N_{\Phi}$ acts as $A \mapsto \Phi^{*} \circ[A,-]$ and $N_{\Phi} f: \Phi^{*}[B,-] \Rightarrow \Phi^{*}[A,-]$ is the natural transformation with components $\Phi^{*}[B, C] \stackrel{\Phi^{*}[f, C]}{\longrightarrow}$ $\Phi^{*}[A, C]$, for any $\mathcal{V}$-morphism $A \stackrel{f}{\rightarrow} B$. Now the natural transformations $\left|N_{\Phi} f\right|:\left|\Phi^{*}[B,-]\right|=$ $\mathcal{V}(B,-) \Rightarrow \mathcal{V}(A,-)=\left|\Phi^{*}[A,-]\right|$ correspond one-to-one to $\mathcal{V}$-morphisms $A \stackrel{f}{\rightarrow} B$, which proves 1 . The first identity of item 2 follows from Equation (11) since $\mathcal{V}$ is commutative, hence all operations are homomorphisms; the second one is obvious, as is item 4.

For every functor $S: \mathcal{V} \rightarrow \operatorname{Coalg}(\mathcal{T}, \mathcal{V})$ with $\|-\| \circ S=i d_{\mathcal{V}}$ there exists by Proposition 20 the algebraic functor $R_{S F_{\mathcal{V} 1}}: \mathcal{V} \rightarrow \operatorname{Alg} \mathcal{T}$. Let $\Phi_{S}: \mathcal{T} \rightarrow \mathcal{T}_{\mathcal{V}}$ be the morphism of Lawvere theories with $\Phi_{S}{ }^{*}=R_{S F_{\mathcal{V} 1}}$. The assignment $S \mapsto \Phi_{S}$ is essentially injective: indeed, for each $\mathcal{V}$-homomorphism $F_{\mathcal{V}} 1 \stackrel{f}{\rightarrow} A$ the $\mathcal{T}$-coalgebra morphism $S f$ has $f$ as its underlying morphism in $\mathcal{V}$ and this satisfies, for each each $\sigma \in \mathcal{T}(n, 1)$, the equation $F_{\mathcal{V}} 1 \stackrel{f}{\rightarrow} A \stackrel{\sigma_{S A}}{\longrightarrow} n \cdot A=F_{\mathcal{V}} 1 \stackrel{\sigma_{S F_{\mathcal{V}} 1}}{\longrightarrow} A \stackrel{n \cdot f}{\longrightarrow} n \cdot A$. This proves that $S$ is determined by $S F_{\mathcal{V}} 1$ since the family $\left(F_{\mathcal{V}} 1 \stackrel{f}{\rightarrow} A\right)_{f \in \mathcal{V}\left(F_{\mathcal{V} 1, A)}\right.}$ is jointly epimorphic. Items 2. and 3. of Theorem 28 imply $R_{N_{\Phi} F_{\mathcal{V}} 1} \simeq \Phi^{*}$ for each $\Phi$. In other words, the assignment $S \mapsto \Phi_{S}$ is essentially bijective.

Theorem 29 Let $\Phi: \mathcal{T} \rightarrow \mathcal{T}_{\mathcal{V}}$ be a theory morphism into a commutative theory $\mathcal{T}_{\mathcal{V}}$. Then the following hold.

1. For every $\mathcal{T}$-coalgebra $\mathrm{A}$ in $\mathcal{V}$ the set $G_{\Phi}(\mathrm{A})$ is the underlying set of a $\mathcal{V}$-subalgebra of $\|\mathrm{A}\|$, such that the functor $G_{\Phi}$ factors as $\operatorname{Coalg}(\mathcal{T}, \mathcal{V}) \stackrel{\bar{G}_{\Phi}}{\longrightarrow} \mathcal{V} \stackrel{|-|}{\longrightarrow}$ Set and the natural transformation e lifts to a natural transformation $\bar{e}: \bar{G}_{\Phi} \Rightarrow\|-\|$. The lifted functor $\bar{G}_{\Phi}$ satisfies the equations $\bar{G}_{\Phi} N_{\Phi}=\mathrm{id} \mathcal{V}$ and $\bar{G}_{\Phi} V_{\Phi}(X)=F_{\mathcal{V}} X$ for each set $X$. 
2. $N_{\Phi}$ is left adjoint to $\bar{G}_{\Phi}$; hence $\mathcal{V}$ is (equivalent to) a full coreflective subcategory of Coalg $(\mathcal{T}, \mathcal{V})$.

Proof Concerning item 1 observe first that $G_{\Phi}(\mathrm{A})$ is the underlying set of a $\mathcal{V}$-subalgebra of $A$ by Equation (12), since the maps $\Phi(\tau)^{n \cdot A}$ are homomorphisms in $\mathcal{V}$. In particular, $G_{\Phi}$ factors over $\mathcal{V}$. The lifted functor $\operatorname{Coalg}(\mathcal{T}, \mathcal{V}) \stackrel{\bar{G}_{\Phi}}{\longrightarrow} \mathcal{V}$ obviously satisfies the equation $\bar{G}_{\Phi} N_{\Phi}=i d_{\mathcal{V}}$. Finally, item 1 implies, again by the lifting theorem of adjunctions, that the functor $\bar{G}_{\Phi}$ has a left adjoint $L$, which then satisfies the equation $L\left(F_{\mathcal{V}} X\right)=V_{\Phi}(X)$. Now $N_{\Phi}\left(F_{\mathcal{V}} 1\right)=L\left(F_{\mathcal{V}} 1\right)$ follows by item 2 of Theorem 28, and this implies $L=N_{\Phi}$ by item 4 of Theorem 28 .

Remark 30 For any theory morphism $\Phi: \mathcal{T} \rightarrow \mathcal{T}_{\mathcal{V}}$ into a commutative theory $\mathcal{T}_{\mathcal{V}}$ the following are equivalent:

1. $N_{\Phi}$ is an equivalence with equivalence inverse $\bar{G}_{\Phi}$.

2. $G_{\Phi} \simeq|-| \circ\|-\|$.

3. $N_{\Phi} \simeq C_{\mathcal{T}, \mathcal{V}}$.

The following examples show that the functors $N_{\Phi}$ may or may not be equivalences.

Examples 31 1. If $\mathcal{V}$ is a commutative variety all of whose algebras are free, then $N$ is an equivalence as is easily seen. By [17] there are essentially four such varieties: Set, the variety of pointed sets $S_{e t}$, Vect $t_{k}$ and $A f f_{k}$, the varieties of vector spaces and affine spaces, respectively, over a field $k$. Thus, if $\mathcal{V}$ is any of these varieties, then every $\mathcal{T}_{\mathcal{V}}$-coalgebra in $\mathcal{V}$ is of the form $\mathcal{V}(X)$ as it is the case for the variety of groups (see Remark 26).

2. By the last example in Section 2.2 there are the equivalences Coalg $\left(A b, \operatorname{Mod}_{R}\right) \simeq \operatorname{Mod}_{R} \simeq$ $\operatorname{Coalg}\left(\operatorname{Grp}, \operatorname{Mod}_{R}\right)$ and $\operatorname{Coalg}\left({ }_{c} \operatorname{Mon}, \operatorname{Mod}_{R}\right) \simeq \operatorname{Mod}_{R} \simeq \operatorname{Coalg}\left(\operatorname{Mon}, \operatorname{Mod}_{R}\right)$, which we will use below. The forgetful functors coincide with $\bar{G}_{\Phi}$ and are equivalences, concrete over ${ }_{c} M o n$ and $A b$, respectively, having the functors $N_{\Phi}$ as their inverses.

3. Let $\mathcal{V}={ }_{c} \mathrm{Sem}$ be the variety of commutative semigroups, and $\Phi^{*}: A b \rightarrow{ }_{c} \mathrm{Sem}$ the forgetful functor. Note that ${ }_{c} \mathrm{Sem}$ is commutative but fails to be semi-additive.

The coproduct $S+T$ in $\mathcal{V}$ is given as $\left(S^{0} \times T^{0}\right) \backslash\{(0,0)\}$ with the component-wise addition, where $S^{0}$ (respectively $T^{0}$ ) denotes the unitarization of $S$ (resp. $T$ ), and with coproduct injections $\nu_{1}(a):=(a, 0), a \in S$, and $\nu_{2}(b):=(0, b), b \in T$. Then $N(S)$ is an internal $\mathcal{T}_{c}$ Sem $^{-}$ coalgebra in ${ }_{c} S e m$ with comultiplication $\mu_{N S}=\mu^{2 \cdot S} \circ\left\langle\nu_{1}, \nu_{2}\right\rangle$, i.e., $\mu_{N S}(a)=(a, 0)+(0, a)=$ $(a, a), a \in S$.

(a) Let $S$ be a commutative semigroup that contains an idempotent element $e$ (e.g. the underlying semigroup of a commutative monoid). Let $E_{e}: S \rightarrow S+S$ be the semigroup homomorphism given by $E_{e}(a):=(e, e), a \in S$. This endows $S$ with a structure of an internal $\mathcal{T}_{c S e m}$-coalgebra in ${ }_{c} S e m$ not isomorphic to that obtained by applying $N$ when $S$ is not reduced to $e$. This shows that $N$ cannot be an equivalence between ${ }_{c} \mathrm{Sem}$ and $\mathrm{Coalg}\left(\mathcal{T}_{c \mathrm{Sem}},{ }_{c} \mathrm{Sem}\right)$.

(b) The comultiplication in $N_{\Phi}(A)$, for an abelian group $A$, thus, is given by $a \mapsto(a, a)$. But $A$ carries also a trivial internal $\mathcal{T}_{c S e m}$-coalgebra structure, namely, $Z_{A}(a):=$ $(0,0)$. This clearly implies that $N_{\Phi}$ is not an equivalence of the categories $A b$ and Coalg $\left(\mathcal{T}_{c S e m}, A b\right)$. 


\subsubsection{Further applications}

Lemma 32 Let $\Phi=\mathcal{T}_{\mathcal{W}} \stackrel{\Xi}{\rightarrow} \mathcal{T}_{\mathcal{U}} \stackrel{\Sigma}{\rightarrow} \mathcal{T}_{\mathcal{V}}$ be a factorization of a morphism of Lawvere theories over a commutative theory $\mathcal{T}_{\mathcal{U}}$. Then the following diagram commutes

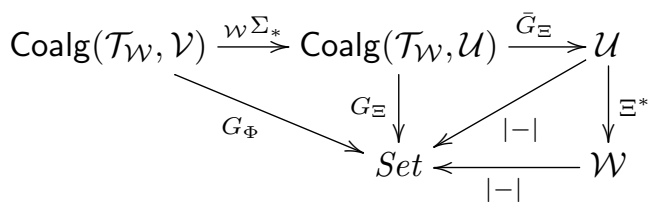

and, moreover,

1. $\bar{G}_{\Xi}\left(\mathcal{W}^{\left.\Sigma_{*} \mathrm{~A}\right)}\right.$ is a $\mathcal{U}$-subalgebra of $\bar{G}_{\Xi}\left(\mathcal{W}^{\Sigma_{*}}\left(C_{\mathcal{T}_{\mathcal{W}}, \mathcal{V}}(\|\mathrm{A}\|)\right)\right)=\bar{G}_{\Xi}\left(C_{\mathcal{T}_{\mathcal{W}}, \mathcal{U}}\left(\Sigma^{*}(\|\mathrm{~A}\|)\right)\right)$ for every $\mathcal{T}_{\mathcal{W}}$-algebra $\mathrm{A}$ in $\mathcal{V}$

2. $\bar{G}_{\Xi}\left(\mathcal{W} \Sigma_{*} \mathrm{~A}\right)$ is a $\mathcal{U}$-subalgebra of $\bar{G}_{\Xi}\left(\left(\Sigma^{*}(\|\mathrm{~A}\|)\right)\right)$, if $\bar{G}_{\Xi}$ is an isomorphism.

Proof The first statement is obvious (see Lemma 24). Concerning the second one note that, with $\mathrm{A} \stackrel{\eta_{\mathrm{A}}}{\longrightarrow}\left\|C_{\mathcal{T}_{\mathcal{W}, \mathcal{V}}}\right\|$ the adjunction unit, the injective map $\left\|\eta_{\mathrm{A}}\right\| \mid \circ e_{\mathrm{A}}$ factors as $e_{C_{\mathcal{T}_{\mathcal{W}}, \mathcal{V}}(\|\mathrm{A}\|)} \circ G_{\Phi}\left(\eta_{\mathrm{A}}\right)$ by naturality (see Equation $12 p)$. This implies that $\bar{G}_{\Xi}\left(\mathcal{W}_{\mathcal{W}} \Sigma_{*}\left(\eta_{\mathrm{A}}\right)\right.$ is a $\mathcal{U}$-monomorphism. Item 3 . follows trivially (see also Remark 30 .

In the discussion of the following applications we use notations as in Diagram (9).

More on primitive elements. $\quad 1$. The primitive element functor ${ }_{c}$ Bialg $_{R} \stackrel{G_{\Phi}}{\longrightarrow}$ Set has by Lemma 32 the following factorization where, by Examples $31 \mid 2, \bar{G}_{\Xi}$ is an isomorphism with inverse $N_{\Xi}$ and, thus, can be identified with the functor $\|-\|$.

$$
\text { Coalg }\left(\operatorname{Mon}_{c}{ }_{c} \operatorname{Alg}_{R}\right) \stackrel{\mathcal{M} \Sigma_{*}}{\longrightarrow} \operatorname{Coalg}\left(\operatorname{Mon}, \operatorname{Mod}_{R}\right) \stackrel{\bar{G}_{\Xi}}{\longrightarrow} \operatorname{Mod}_{R} \stackrel{|-|}{\longrightarrow} \text { Set. }
$$

We so obtain the following familiar result. For each commutative Hopf algebra $A$ the set $G_{\Phi}(A)$ of primitive elements of $A$ is a submodule of the the carrier algebra $\Sigma^{*}(A)$.

2. Recall that each symmetric algebra, that is, each free commutative $R$-algebra $\Sigma_{*}(M)$ over an $R$-module $M$, carries the structure of a Hopf algebra $S(M)$, where $\operatorname{Mod}_{R} \stackrel{\Xi^{*}}{\longrightarrow}$ $A b$ is the forgetful functor. This construction is given by the functor

$$
S=\operatorname{Mod}_{R} \stackrel{N_{\Xi}}{\longrightarrow} \operatorname{Coalg}\left(A b, \operatorname{Mod}_{R}\right) \stackrel{\mathcal{W}_{\Sigma_{*}}}{\longrightarrow} \operatorname{Coalg}\left(A b,{ }_{c} A l g_{R}\right) \simeq{ }_{b i} H_{o p f}
$$

$S$ is left adjoint to $G_{\Xi} \circ \mathcal{W}^{\Sigma_{*}}$ by Proposition 16 , since $N_{\Xi}$ has $G_{\Xi}$ as its inverse (see Examples 31), this adjunction is essentially the adjunction $\mathcal{W}_{\Sigma_{*}} \dashv \mathcal{W} \Sigma_{*}$. Since the unit of this adjunction is point-wise monomorphic (see the proof of Proposition 16), we obtain the familiar result that the set of primitive elements of $S(M)$ contains $M$.

More on group-like elements. Since Coalg $\left(\right.$ Mon,${ }_{c}$ Mon $)=\operatorname{Coalg}\left({ }_{c}\right.$ Mon,${ }_{c}$ Mon $)$ (see Examples 31|2), the group-like element functor ${ }_{c}$ Bialg $_{R} \stackrel{G_{\Psi}}{\longrightarrow}$ Set factors by Lemma 32 as

$$
\text { Coalg }\left(\text { Mon },{ }_{c} A l g_{R}\right) \stackrel{\mathcal{\tau}_{\mathcal{M}}{ }^{\Psi_{*}}}{\longrightarrow} \text { Coalg }\left(\text { Mon },{ }_{c} \text { Mon }\right) \stackrel{\bar{G}_{c \mathcal{M}}}{\longrightarrow}{ }_{c} \text { Mon } \stackrel{|-|}{\longrightarrow} \text { Set. }
$$

where the functor $\bar{G}_{c} \mathcal{M}$ is an isomorphism. We so obtain the fact that the set of grouplike elements of a commutative bialgebra (hence, in particular, of any commutative Hopf algebra) $A$ is a submonoid of the multiplicative monoid of the underlying algebra of $A$. 


\section{References}

[1] Abe, E., Hopf algebras, Cambridge University Press (1977).

[2] Adámek, J. and Rosický, J., Locally Presentable and Accessible Categories, Cambridge University Press, Cambridge (1994).

[3] Adámek, J. and Rosický, J., On sifted colimits and generalized varieties, Theory and Applications of Categories 8 (2001), 33-53.

[4] Adámek, J., Rosický, J. and Vitale, E.M., Algebraic theories, Cambridge University Press, Cambridge (2011).

[5] Aguiar, M., A note on strongly separable algebras, Bol. Acad. Nac. Cienc. (Córdoba) 65 (2000), 51-60.

[6] Anderson, W.A. and Fuller, K.R., Rings and Categories of Modules, Springer, New YorkHeidelberg-Berlin (1974).

[7] Bergman, G.M., Colimits of representable algebra-valued functors, Theory Appl. Categ. 20 (2008), 334-404.

[8] Bergman, G.M., An Invitation to General Algebra and Universal Constructions, 2nd edition, Springer International Publishing Switzerland (2015).

[9] Bird, G.J., Limits in 2-categories of locally presentable categories, $P h D$ thesis, University of Sidney (1984).

[10] Borceux, F., Handbook of Categorical Algebra 2, Cambridge University Press, Cambridge (1994).

[11] Brzezinski, T. and Wisbauer, R., Corings and Comodules. Lond. Math. Soc. Lect. Note Ser. 309, Cambridge University Press, Cambridge (2003).

[12] Davey, B. A. and Davis, G., Tensor products and entropic varieties, Algebra Universalis 21 (1985), 68-88.

[13] Davis, R., Free coalgebras in a category of rings, Proc. Amer. Math. Soc. 25 (1970), 155-158; Review MR0258712.

[14] Eilenberg, S., Abstract description of some basic functors, J. Ind. Math. Soc. 24 (1960), $231-234$

[15] Eilenberg, S. and Moore, J. C., Adjoint functors and triples, Illinois J. Math. 9 (1965), 381-398.

[16] Freyd, P., Algebra valued functors in general and tensor products in particular, Colloq. Math. (Warszawa) 14 (1966), 89-106; Review Zbl 0144.01003.

[17] Givant, S., Universal Horn classes categorical or free in power, Annals of Mathematical Logic 15 (1979), 1-53.

[18] Isbell, J.R., General functorial semantics, American Journal of Mathematics 94, (1972), 535-596 
[19] Kan, D.M., On monoids and their duals, Bol. Soc. Mat. Mexicana (2), 3 (1958), 52-61.

[20] Lawvere, F.W., Functorial semantics of algebraic theories and some algebraic problems in the context of functorial semantics of algebraic theories, $\mathrm{PhD}$ Thesis, Columbia University (1963), Reprinted as Reprints in Theory and Applications of Categories No. 5 (2004), 1-121.

[21] Linton, F.E.J., Some aspects of equational categories, in: Proc. Conf. Cat. Algebra La Jolla 1965, Springer, New York 1966, 84-94

[22] Mac Lane, S., Categories for the Working Mathematician, 2nd ed., Springer, New York (1998).

[23] Nyman, A. and Smith, S. P., A generalization of Watts's theorem: right exact functors on module categories, Communications in Algebra, 44:7 (2016), 3160-3170.

[24] Popescu, N. Abelian Categories with Applications to Rings and Modules, Academic Press, London (1973).

[25] Porst, H.-E., Equivalence for varieties in general and for BOOL in particular, Algebra univers. 43 (2000), $157-186$

[26] Porst, H.-E., On subcategories of the category of Hopf algebras, Arab. J. Sci. Eng. 36 (2011), $1023-1029$

[27] Porst, H.-E., Free internal groups, Appl. Categor. Struct. 20 (2012), 31—42.

[28] Tall, D. O. and Wraith, G. C., Representable functors and operations on rings, Proc. London Math. Soc. 20 (1970), 619-643

[29] Watts, C. E., Intrinsic characterizations of some additive functors, Proc. Amer. Math. Soc. 11 (1960), 5-8. 